\title{
Article
}

\section{Categorical Aspects of Quantum Groups: Multipliers and Intrinsic Groups}

\author{
Daws, Matthew
}

Available at https://clok.uclan.ac.uk/23496/

Daws, Matthew orcid iconORCID: 0000-0003-1707-4308 (2018) Categorical Aspects of Quantum Groups: Multipliers and Intrinsic Groups. Canadian Journal of Mathematics, 68 (2). pp. 309-333. ISSN 0008-414X

It is advisable to refer to the publisher's version if you intend to cite from the work. http://dx.doi.org/10.4153/CJM-2015-022-0

For more information about UCLan's research in this area go to http://www.uclan.ac.uk/researchgroups/ and search for < name of research Group>.

For information about Research generally at UCLan please go to http://www.uclan.ac.uk/research/

All outputs in CLoK are protected by Intellectual Property Rights law, including Copyright law. Copyright, IPR and Moral Rights for the works on this site are retained by the individual authors and/or other copyright owners. Terms and conditions for use of this material are defined in the policies page.

\section{CLoK}

Central Lancashire online Knowledge www.clok.uclan.ac.uk

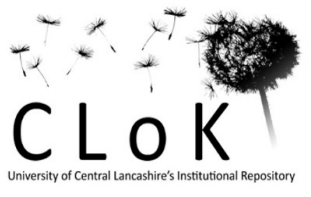




\title{
Categorical aspects of quantum groups: multipliers and intrinsic groups
}

\author{
Matthew Daws
}

January 22, 2018

\begin{abstract}
We show that the assignment of the (left) completely bounded multiplier algebra $M_{c b}^{l}\left(L^{1}(\mathbb{G})\right)$ to a locally compact quantum group $\mathbb{G}$, and the assignment of the intrinsic group, form functors between appropriate categories. Morphisms of locally compact quantum groups can be described by Hopf $*$-homomorphisms between universal $C^{*}$-algebras, by bicharacters, or by special sorts of coactions. We show that the whole theory of completely bounded multipliers can be lifted to the universal $C^{*}$-algebra level, and that then the different pictures of both multipliers (reduced, universal, and as centralisers) and morphisms interact in extremely natural ways. The intrinsic group of a quantum group can be realised as a class of multipliers, and so our techniques immediately apply. We also show how to think of the intrinsic group using the universal $C^{*}$-algebra picture, and then, again, show how the differing views on the intrinsic group interact naturally with morphisms. We show that the intrinsic group is the "maximal classical" quantum subgroup of a locally compact quantum group, show that it is even closed in the strong Vaes sense, and that the intrinsic group functor is an adjoint to the inclusion functor from locally compact groups to quantum groups.

Keywords: Locally compact quantum group, morphism, intrinsic group, multiplier, centraliser.

2010 Mathematics Subject Classification: Primary 20G42, 46L89; Secondary 22D25, 43A22, 43A35, 43A95, 46L52, 47L25
\end{abstract}

\section{Introduction}

Locally compact quantum groups, [20], are now recognised as the correct way to think about quantum groups from an operator algebraic viewpoint; or alternatively, as a non-commutative extension of Pontryagin Duality, building on the theory of Kac Algebras and Multiplicative Unitary theory. Thinking from a category theory perspective, it is also extremely interesting to think about what the morphisms of such objects should be. The recent paper [24], building on [25, 17], gives a very satisfactory answer.

As already seen for a non-amenable group $G$, when forming the group algebra we can either look at the reduced case $C_{r}^{*}(G)$ or the universal case $C^{*}(G)$, which are different. The same applies to a quantum group $\mathbb{G}$ giving, in general different, algebras $C_{0}(\mathbb{G})$ and $C_{0}^{u}(\mathbb{G})$. Morphisms can either be expressed as Hopf $*$-homomorphisms, at the universal algebra level, or through bicharacters, or special types of coaction, at the reduced algebra level (or equivalently at the von Neumann algebra level, compare [17, Section 12]). This gives further weight to the view that $C_{0}(\mathbb{G})$ and $C_{0}^{u}(\mathbb{G})$ are just different facets of the same "quantum group" $\mathbb{G}$.

There has been much interest recently in studying completely bounded multipliers of $\mathbb{G}$, or more precisely, of the convolution algebra $L^{1}(\mathbb{G})$, see [7, 8, 13, 14] for example. This is an $L^{1}(\mathbb{G})$ theory, or equivalently an $L^{\infty}(\mathbb{G})$ theory. In this paper we show how to cast the entire theory using $C_{0}^{u}(\mathbb{G})$, again showing that the distinction between $C_{0}(\mathbb{G})$ and $C_{0}^{u}(\mathbb{G})$ is in many ways unimportant. We believe that, even for completely bounded multipliers of the Fourier algebra, this approach, that is, using $C^{*}(G)$ and not $V N(G)$, has not previously been studied.

Using this theory, in terms of universal algebras, leads to a simple way to study how multipliers and morphisms interact, generalising the study Spronk undertook of cb multipliers of $A(G)$ in $[27$, 
Section 6.1]. We then find that all the different "pictures" of morphisms have a very natural interpretation with the different aspects of multipliers (essentially, whether one works with centralisers, multipliers, or multipliers at the $C_{0}^{u}(\mathbb{G})$ level).

The second topic we look at is that of intrinsic groups, [16, 5]. We can view the intrinsic group as an assignment, to each quantum group, of a group. Following [16] we can define the intrinsic group using multipliers, and thus apply our previous study of morphisms to multipliers. We use this to show that the intrinsic group is a functor, and again show that the different pictures of the intrinsic group, and the different pictures of morphisms, interact very naturally. Again, here it is actually most natural to work with $C_{0}^{u}(\widehat{\mathbb{G}})$. We give the new result that the intrinsic group of $M\left(C_{0}^{u}(\widehat{\mathbb{G}})\right)$, with the relative strict topology, forms another natural equivalent definition of the intrinsic group of $\mathbb{G}$.

Let us say that $\mathbb{G}$ is "classical" if $C_{0}(\mathbb{G})=C_{0}(G)$ for a locally compact group $G$. We show that the intrinsic group is the maximal "classical subgroup" of a quantum group. Closed subgroups of quantum groups were explored in [10], with two definitions offered. We show that the intrinsic group is closed in the stronger, "Vaes closed" sense. We also recast this maximality property as a universal property in the category theory sense, and show the the intrinsic group functor is the adjoint of the inclusion functor from the category of locally compact groups to the category of locally compact quantum groups. A corollary of these results is that any classical, closed quantum subgroup is Vaes closed, a result not available from [10].

Briefly, the organisation of the paper is as follows. We firstly introduce locally compact quantum groups, and then summarise the notion of a "morphism". Compared to [24] we work with "left" multiplicative unitaries, and also "reverse the arrows", to better generalise from classical group homomorphisms. As such, we take a little time to give a detailed summary, in the hope that this will be a useful reference. In Section 3 we introduce centralisers and multipliers, and show how to work with $C_{0}^{u}(\mathbb{G})$ instead of $L^{\infty}(\mathbb{G})$. We then apply this theory to show how multipliers and morphisms interact, finishing with some comments on operator space structures and making links with the results of [14. In Section 4 we introduce the intrinsic group, again lift the theory to $C_{0}^{u}(\mathbb{G})$ (or actually the multiplier algebra, with the strict topology). This allows us to streamline some of the proofs from [16]. Then in Section 5 we show that the intrinsic group is a functor, show the stated universal property, and then look at closed quantum subgroups.

\subsection{Acknowledgements}

We would like to thank Matthias Neufang and Sutanu Roy for showing an interest in this work at an early stage, and for asking about adjoint functors. We thank Stefaan Vaes for bringing the appendix of [2] to our attention. We thank the referee for useful comments.

\section{Locally compact quantum groups and their morphisms}

Let us introduce some notation. We write $\otimes$ for the minimal tensor product of $C^{*}$-algebras or the tensor product of Hilbert spaces, and write $\bar{\otimes}$ for the normal tensor product of von Neumann algebras. For a $C^{*}$-algebra $A$, we write $M(A)$ for the multiplier algebra. We shall use the theory of non-degenerate $*$-homomorphisms, and their strict extensions, see [21] or the appendix of [23] for example. We shall write $\mathcal{B}(H)$ for the bounded linear operators on a Hilbert space $H$, and write $\mathcal{B}_{0}(H)$ for the compact operators. We use the standard theory of slice maps, and the "leg numbering notation". We shall use $\sigma$ to denote the tensor swap map, acting on Hilbert spaces or algebras. 
We shall use basic, standard results from the theory of Operator Spaces, specifically the notion of a Completely Bounded map, see [11].

For the theory of locally compact quantum groups, we use [20, 19]. For more gentle introductions, see [18, 29], and compare also [23]. We follow the now standard notation, and for a locally compact quantum group $\mathbb{G}$ write $C_{0}(\mathbb{G})$ for the $C^{*}$-algebra representing $\mathbb{G}$, and $L^{\infty}(\mathbb{G})$ for the von Neumann algebra, with $L^{1}(\mathbb{G})$ its predual. Writing $\Delta$ for the coproduct, we can either view $\Delta$ as a non-degenerate $*$-homomorphism $C_{0}(\mathbb{G}) \rightarrow M\left(C_{0}(\mathbb{G}) \otimes C_{0}(\mathbb{G})\right.$ ), or as a unital injective normal *-homomorphism $L^{\infty}(\mathbb{G}) \rightarrow L^{\infty}(\mathbb{G}) \bar{\otimes} L^{\infty}(\mathbb{G})$, which thus induces an algebra structure on $L^{1}(\mathbb{G})$. Similarly, $\Delta$ induces an algebra product on $C_{0}(\mathbb{G})^{*}$. We shall denote these products by $\star$.

Locally compact quantum groups carry, by definition, invariant weights. Use the left invariant weight to form the Hilbert space $L^{2}(\mathbb{G})$. Then there is the fundamental multiplicative unitary $W$ on $L^{2}(\mathbb{G}) \otimes L^{2}(\mathbb{G})$, which implements the coproduct as $\Delta(x)=W^{*}(1 \otimes x) W$ for $x \in L^{\infty}(\mathbb{G})$. Then $C_{0}(\mathbb{G})$ is weak*-dense in $L^{\infty}(\mathbb{G})$ and the inclusion $C_{0}(\mathbb{G}) \rightarrow L^{\infty}(\mathbb{G})$ repsects all of the associated maps. One can start at either the $C^{*}$-algebra level, [20], or at the von Neumann algebra level, [19]. The set $\left\{(\omega \otimes \mathrm{id})(W): \omega \in \mathcal{B}\left(L^{2}(G)\right)_{*}\right\} \subseteq \mathcal{B}\left(L^{2}(\mathbb{G})\right)$ is an algebra, and its closure a $C^{*}$-algebra, $C_{0}(\widehat{\mathbb{G}})$. We can introduce a coproduct on $C_{0}(\widehat{\mathbb{G}})$ by $\widehat{\Delta}(x)=\widehat{W}^{*}(1 \otimes x) \widehat{W}$, where $\widehat{W}=\sigma(W)^{*}$. Then $C_{0}(\widehat{\mathbb{G}})$ can be given invariant weights so as to become a locally compact quantum group, the weak*-closure is $L^{\infty}(\widehat{\mathbb{G}})$ which is the von Neumann algebraic version of $C_{0}(\widehat{\mathbb{G}})$, and so we obtain $\widehat{\mathbb{G}}$. Then $W \in M\left(C_{0}(\mathbb{G}) \otimes C_{0}(\widehat{\mathbb{G}})\right) \subseteq L^{\infty}(\mathbb{G}) \bar{\otimes} L^{\infty}(\widehat{\mathbb{G}})$.

The algebras $C_{0}(\mathbb{G})$ and $L^{\infty}(\mathbb{G})$ admit further maps, such as the antipode, the unitary antipode, and the scaling group, but these will not play a prominent role in this paper.

For a classical group $G$, we can form $C_{0}(G)$ and $C_{r}^{*}(G)$. Alternatively, we might form the universal group $C^{*}$-algebra $C^{*}(G)$. There is a quantum group analogue of this, [17]. We shall write $C_{0}^{u}(\mathbb{G})$ for the "universal version" of $C_{0}(\mathbb{G})$; it is the enveloping $C^{*}$-algebra of a certain *-subalgebra of $L^{1}(\widehat{\mathbb{G}})$. We can lift $\Delta$, the antipode, and so forth, to $C_{0}^{u}(\mathbb{G})$. We shall continue to write $\Delta$ for the coproduct on $C_{0}^{u}(\mathbb{G})$. There is a quotient $*$-homomorphism $\pi: C_{0}^{u}(\mathbb{G}) \rightarrow C_{0}(\mathbb{G})$, the "reducing morphism", which intertwines all of the associated maps. Finally, we can lift $W$ to various universal versions. Here we depart from the notation of [17] and write $\mathbb{W} \in M\left(C_{0}^{u}(\mathbb{G}) \otimes C_{0}^{u}(\widehat{\mathbb{G}})\right)$ for what is denoted by $\mathcal{U}$ in [17], and then let $\mathbb{W}=(\mathrm{id} \otimes \widehat{\pi})(\mathbb{W})$ and $\mathbb{W}=(\pi \otimes \mathrm{id})(\mathbb{W})$, which are denoted by $\mathcal{V}, \widehat{\mathcal{V}}$, respectively, in [17]. An important result for us is that

$$
(\mathrm{id} \otimes \pi) \Delta(a)=\mathbb{W}^{*}(1 \otimes \pi(a)) \mathbb{W} \quad\left(a \in C_{0}^{u}(\mathbb{G})\right),
$$

see [17, Proposition 6.2].

As indicated here, when dealing with objects associated to $\widehat{\mathbb{G}}$, we shall adorn the object with a hat. When dealing with more than one quantum group, we shall adorn the objects with $\mathbb{G}$ or $\mathbb{H}$, and so forth, as appropriate. For example, $W_{\mathbb{H}}$ is the fundamental unitary associated with $\mathbb{H}$, and we have that $\widehat{W}_{\mathbb{G}}=W_{\widehat{\mathbb{G}}}$. Note that in the notation of [17], we have that $\widehat{\mathcal{V}}_{\mathbb{G}}=\sigma\left(\mathcal{V}_{\widehat{\mathbb{G}}}\right)^{*}$, which explains our use of different notation.

Finally, we remark that a very similar theory can be built from just working with special ("manageable" or "modular") multiplicative unitaries, see [31, 26]. All of the results of this paper, excepting Section 5.2, hold in this more general setting.

\subsection{Morphisms of quantum groups}

A morphism $\mathbb{G} \rightarrow \mathbb{H}$ can be described in a number of equivalent ways, [24, 25]. We shall work with "left" bicharacters, so as to more closely match the conventions of Kustermans and Vaes. We shall also "reverse the arrows", as compared to [24], so that a group homomorphism $G \rightarrow H$ will give rise a morphism $\mathbb{G} \rightarrow \mathbb{H}$ if $C_{0}(\mathbb{G})=C_{0}(G)$ and $C_{0}(\mathbb{H})=C_{0}(H)$ and not the other way around. 
As such, we shall give more detail in this summary than strictly necessary, in the hope it will be a useful reference.

A morphism $\mathbb{G} \rightarrow \mathbb{H}$ can be equivalently described as:

1. A non-degenerate $*$-homomorphism $\phi: C_{0}^{u}(\mathbb{H}) \rightarrow M\left(C_{0}^{u}(\mathbb{G})\right)$ which intertwines the coproducts. That is, a Hopf *-homomorphism between universal quantum groups.

2. As a bicharacter, which is a unitary $U \in M\left(C_{0}(\mathbb{G}) \otimes C_{0}(\widehat{\mathbb{H}})\right)$ which satisfies $\left(\Delta_{\mathbb{G}} \otimes \mathrm{id}\right)(U)=$ $U_{13} U_{23}$ and $\left(\mathrm{id} \otimes \Delta_{\widehat{\mathbb{H}}}\right)(U)=U_{13} U_{12}$.

3. As a special type of coaction, termed a left quantum group homomorphism, which is a nondegenerate $*$-homomorphism $\beta: C_{0}(\mathbb{H}) \rightarrow M\left(C_{0}(\mathbb{G}) \otimes C_{0}(\mathbb{H})\right)$ which is a coaction of $\mathbb{G}$, namely $\left(\Delta_{\mathbb{G}} \otimes \mathrm{id}\right) \beta=(\mathrm{id} \otimes \beta) \beta$, and which also satisfies $\left(\mathrm{id} \otimes \Delta_{\mathbb{H}}\right) \beta=(\beta \otimes \mathrm{id}) \Delta_{\mathbb{H}}$.

Alternatively, we can express the second two conditions at the von Neumann algebra level:

1. $U \in L^{\infty}(\mathbb{G}) \bar{\otimes} L^{\infty}(\widehat{\mathbb{H}})$ unitary with the same conditions;

2. $\beta: L^{\infty}(\mathbb{H}) \rightarrow L^{\infty}(\mathbb{G}) \bar{\otimes} L^{\infty}(\mathbb{H})$ a normal unital $*$-homomorphism, with the same conditions.

This can be shown by adapting the proofs of [24]; compare also the different proofs in [17, Section 12]. These different notions are linked by the following properties:

1. Given $\phi$, we have that $U=\left(\pi_{\mathbb{H}} \phi \otimes \mathrm{id}\right)\left(\mathbb{W}_{\mathbb{H}}\right)$ and $\beta$ is the unique $*$-homomorphism which satisfies that $\beta \pi_{\mathbb{H}}=\left(\pi_{\mathbb{G}} \phi \otimes \pi_{\mathbb{H}}\right) \Delta_{\mathbb{H}}^{u}$.

2. Given $U$, we define $\beta$ by $\beta(x)=U^{*}(1 \otimes x) U$ for $x \in C_{0}(\mathbb{H})$ or $L^{\infty}(\mathbb{H})$. We can always "lift" $U$ to a bicharacter in $M\left(C_{0}^{u}(\mathbb{G}) \otimes C_{0}^{u}(\widehat{\mathbb{H}})\right)$ and then the results of [17, Section 6] readily give a unique $\phi$ with $U=\left(\pi_{\mathbb{G}} \phi \otimes \mathrm{id}\right)\left(\mathbb{W}_{\mathbb{H}}\right)$.

3. Given $\beta$, there is a unique unitary $U$ with $(\beta \otimes \mathrm{id})\left(W_{\mathbb{H}}\right) W_{\mathbb{H}, 23}^{*}=U_{13}$, and $U$ is a bicharacter. From $U$ we obtain $\phi$, but there appears to be no simple, direct way to move from $\beta$ to $\phi$.

We remark that we also have the notion of a right quantum group homomorphism, but one can easily move between the left and right cases by using the unitary antipodes of $\mathbb{G}$ and $\mathbb{H}$. Furthermore, 24] also explores a fourth equivalent notion, namely that of a certain class of functors between the categories of $C^{*}$-algebras with $C_{0}(\mathbb{G})$-coactions and $C_{0}(\mathbb{H})$-coactions.

The identity morphism $\mathbb{G} \rightarrow \mathbb{G}$ is associated to the bicharacter $W_{\mathbb{G}}$ and the quantum group homomorphism $\Delta_{\mathbb{G}}$. We also remark that (left) quantum group homomorphisms $\beta$ are also injective, and "continuous", meaning that the linear span of $\beta\left(C_{0}(\mathbb{H})\right)\left(C_{0}(\mathbb{G}) \otimes 1\right)$ is contained in, and dense in, $C_{0}(\mathbb{G}) \otimes C_{0}(\mathbb{H})$.

Given a morphism $f: \mathbb{G} \rightarrow \mathbb{H}$, write $\phi_{f}, U_{f}, \beta_{f}$ for the associated objects above. Given $f: \mathbb{G} \rightarrow$ $\mathbb{H}$ and $g: \mathbb{H} \rightarrow \mathbb{K}$, the composition $g f: \mathbb{G} \rightarrow \mathbb{K}$ is associated to:

- $\phi: C_{0}^{u}(\mathbb{K}) \rightarrow M\left(C_{0}^{u}(\mathbb{G})\right)$ given by $\phi=\phi_{f} \circ \phi_{g}$ (the usual composition of non-degenerate *-homomorphisms).

- $U \in M\left(C_{0}(\mathbb{G}) \otimes C_{0}(\widehat{\mathbb{K}})\right)$ which is the unique unitary satisfying $U_{g, 23} U_{f, 12}=U_{f, 12} U_{13} U_{g, 23}$ in $\mathcal{B}\left(L^{2}(\mathbb{G}) \otimes L^{2}(\mathbb{H}) \otimes L^{2}(\mathbb{K})\right)$.

- $\beta: C_{0}(\mathbb{K}) \rightarrow M\left(C_{0}(\mathbb{G}) \otimes C_{0}(\mathbb{K})\right)$ which is the unique non-degenerate $*$-homomorphism satisfying that $\left(\beta_{f} \otimes \mathrm{id}\right) \beta_{g}=\left(\mathrm{id} \otimes \beta_{g}\right) \beta$.

We remark that it is possible to prove the existence of $U$ just using $U_{f}, U_{g}$, and similarly construct $\beta$ just using $\beta_{f}, \beta_{g}$, without having to pass through the various equivalences.

Finally, we mention duality. For any morphism $\mathbb{G} \rightarrow \mathbb{H}$, there is a "dual morphism" $\mathbb{H} \rightarrow \mathbb{G}$. Writing $\phi, U$ for $\mathbb{G} \rightarrow \mathbb{H}$ and $\widehat{\phi}, \widehat{U}$ for $\mathbb{H} \rightarrow \mathbb{G}$, we have that: 
- $\widehat{U}=\sigma\left(U^{*}\right)$ where again $\sigma$ is the swap map;

- $\phi$ and $\widehat{\phi}$ are linked by the relation that $(\phi \otimes \mathrm{id})\left(\mathbb{W}_{\mathbb{H}}\right)=(\mathrm{id} \otimes \widehat{\phi})\left(\mathbb{W}_{\mathbb{G}}\right)$.

We remark that there appears to be no direct way to express the duality relation at the level of coactions. This is perhaps not surprising, as duality is governed by the fundamental unitary, which is reflected in the bicharacter picture, and of course used directly to relate $\phi$ and $\widehat{\phi}$.

\section{Multipliers and morphisms}

Completely bounded multipliers of locally compact quantum groups have been studied in detail in [14, 13] and by the author in [7, 8]. To be careful, we shall follow the notation of [14], but the $C^{*}$-algebraic approach of [8] will pay off here.

Fix a locally compact quantum group $\mathbb{G}$. A left centraliser of $L^{1}(\mathbb{G})$ is a right module map, that is, $L_{*}: L^{1}(\mathbb{G}) \rightarrow L^{1}(\mathbb{G})$ with $L_{*}\left(\omega_{1} \star \omega_{2}\right)=L_{*}\left(\omega_{1}\right) \star \omega_{2}$. Let $C_{c b}^{l}\left(L^{1}(\mathbb{G})\right)$ be the space of all completely bounded left centralisers. For $L_{*} \in C_{c b}^{l}\left(L^{1}(\mathbb{G})\right)$ let $L=\left(L_{*}\right)^{*} \in \mathcal{C B}\left(L^{\infty}(\mathbb{G})\right)$ be the adjoint; that $L_{*}$ is a left centraliser is then equivalent to $L$ being left covariant, $(L \otimes \mathrm{id}) \Delta=\Delta L$.

Recall the left regular representation $\lambda: L^{1}(\mathbb{G}) \rightarrow C_{0}(\widehat{\mathbb{G}})$, an injective algebra homomorphism defined by $\lambda(\omega)=(\omega \otimes \mathrm{id})(W)$. We say that $x \in L^{\infty}(\widehat{\mathbb{G}})$ is a left completely bounded multiplier if $x \lambda(\omega) \in \lambda\left(L^{1}(\mathbb{G})\right)$ for each $\omega \in L^{1}(\mathbb{G})$, and the resulting map $L^{1}(\mathbb{G}) \rightarrow L^{1}(\mathbb{G})$ is completely bounded. Denote this by $x \in M_{c b}^{l}\left(L^{1}(\mathbb{G})\right)$. By definition, $M_{c b}^{l}\left(L^{1}(\mathbb{G})\right) \subseteq C_{c b}^{l}\left(L^{1}(\mathbb{G})\right)$, and a major result of [14], see Corollary 4.4 of that paper, is that every $L_{*} \in C_{c b}^{l}\left(L^{1}(\mathbb{G})\right)$ arises in this way. We finessed this result in $\left[8\right.$, Theorem 4.2], showing that actually $M_{c b}^{l}\left(L^{1}(\mathbb{G})\right) \subseteq M\left(C_{0}(\mathbb{G})\right)$; compare the self-contained approach of [7, Proposition 3.1]. We shall say that $x \in M_{c b}^{l}\left(L^{1}(\mathbb{G})\right)$ is "associated to" $L_{*} \in C_{c b}^{l}\left(L^{1}(\mathbb{G})\right)$. Notice that clearly $M_{c b}^{l}\left(L^{1}(\mathbb{G})\right)$ and $C_{c b}^{l}\left(L^{1}(\mathbb{G})\right)$ are isomorphic algebras under this identification.

In this paper we shall work with left multipliers, but it is easy to see that analogous results hold for right multipliers, either compare [14, 8], or just work with the opposite quantum group, to use the terminology of [19, Section 4]. We remark that double multipliers seem somewhat less well understood, compare [8, Section 7].

\subsection{Moving to the universal level}

In this section, we prove analogous results about the interaction of $C_{c b}^{l}\left(L^{1}(G)\right)$ and $C_{0}^{u}(\widehat{\mathbb{G}})$, in place of $C_{0}(\widehat{\mathbb{G}})$. This then allows us to study how morphisms and multipliers interact, as morphisms are most naturally studied at the level of Hopf $*$-homomorphisms between universal $C^{*}$-algebraic quantum groups. The techniques here are inspired by [8], though here we shall not work with Hilbert $C^{*}$-modules (but one could construct analogous proofs using this machinery).

Let $L_{*} \in C_{c b}^{l}\left(L^{1}(\mathbb{G})\right)$. We wish to consider $(L \otimes \mathrm{id})(\mathrm{W})$, but we need to make sense of this. Let $C_{0}^{u}(\widehat{\mathbb{G}})$ be faithfully and non-degenerated represented on a Hilbert space $K$, so we may identify $M\left(C_{0}^{u}(\widehat{\mathbb{G}})\right)$ with those $x \in \mathcal{B}(K)$ which multiply $C_{0}^{u}(\widehat{\mathbb{G}})$ into itself; then $x \in C_{0}^{u}(\widehat{\mathbb{G}})^{\prime \prime}$. Similarly, $M\left(C_{0}(\mathbb{G}) \otimes C_{0}^{u}(\widehat{\mathbb{G}})\right) \subseteq L^{\infty}(\mathbb{G}) \bar{\otimes} C_{0}^{u}(\widehat{\mathbb{G}})^{\prime \prime} \subseteq \mathcal{B}\left(L^{2}(\mathbb{G}) \otimes K\right)$. Then $(L \otimes \mathrm{id})(\mathrm{W}) \in L^{\infty}(\mathbb{G}) \bar{\otimes} C_{0}^{u}(\widehat{\mathbb{G}})^{\prime \prime}$ is well-defined.

Lemma 3.1. With the notation above, we have that $(L \otimes \mathrm{id})(\mathrm{W}) \in M\left(\mathcal{B}_{0}\left(L^{2}(\mathbb{G})\right) \otimes C_{0}^{u}(\widehat{\mathbb{G}})\right)$.

Proof. By the structure of normal completely bounded maps and the structure theory of normal $*-$ homomorphisms, we can find a Hilbert space $H$ and $S, T \in \mathcal{B}\left(L^{2}(\mathbb{G}), L^{2}(\mathbb{G}) \otimes H\right)$ with $\|S\|\|T\|=$ 
$\|L\|_{c b}$ and with $L(x)=S^{*}(x \otimes 1) T$ for all $x \in L^{\infty}(\mathbb{G})$. See, for example, the discussion in [7, Section 3] or the proof of [8, Theorem 4.2].

Let $\theta \in \mathcal{B}_{0}\left(L^{2}(\mathbb{G})\right)$ and $a \in C_{0}^{u}(\widehat{\mathbb{G}})$. Let $U: L^{2}(\mathbb{G}) \rightarrow L^{2}(\mathbb{G}) \otimes H$ be some isometry, and set $R=T \theta U^{*}$ so that $R U=T \theta$. Then, working in $L^{\infty}(\mathbb{G}) \bar{\otimes} C_{0}^{u}(\widehat{\mathbb{G}})^{\prime \prime} \subseteq \mathcal{B}\left(L^{2}(\mathbb{G}) \otimes K\right)$,

$$
(L \otimes \mathrm{id})(\mathrm{W})(\theta \otimes a)=(S \otimes 1)^{*} \mathrm{~W}_{13}(T \theta \otimes a)=(S \otimes 1)^{*} \mathrm{~W}_{13}(R \otimes a)(U \otimes 1) .
$$

Now, $R \in \mathcal{B}_{0}\left(L^{2}(\mathbb{G}) \otimes H\right)$ because $\theta$ is compact. As $W \in M\left(\mathcal{B}_{0}\left(L^{2}(\mathbb{G})\right) \otimes C_{0}^{u}(\widehat{\mathbb{G}})\right)$, and using that $\mathcal{B}_{0}\left(L^{2}(\mathbb{G}) \otimes H\right)=\mathcal{B}_{0}\left(L^{2}(\mathbb{G})\right) \otimes \mathcal{B}_{0}(H)$, it follows that

$$
\mathrm{W}_{13}(R \otimes a) \in \mathcal{B}_{0}\left(L^{2}(\mathbb{G}) \otimes H\right) \otimes C_{0}^{u}(\widehat{\mathbb{G}}) .
$$

Hence $(L \otimes \mathrm{id})(\mathrm{W})(\theta \otimes a) \in \mathcal{B}_{0}\left(L^{2}(\mathbb{G})\right) \otimes C_{0}^{u}(\widehat{\mathbb{G}})$. Analogously we can show this with the order of products reversed, and so $(L \otimes \mathrm{id})(\mathrm{W}) \in M\left(\mathcal{B}_{0}\left(L^{2}(\mathbb{G})\right) \otimes C_{0}^{u}(\widehat{\mathbb{G}})\right)$ as claimed.

We shall use the "invariants are constant" idea from [24], compare [7, Theorem 2.1] and [1, Lemma 4.6]: if $x, y \in L^{\infty}(\mathbb{G})$ with $\Delta(x)=y \otimes 1$ then $x=y \in \mathbb{C} 1$. In the following, $\lambda_{u}: L^{1}(\mathbb{G}) \rightarrow$ $C_{0}^{u}(\widehat{\mathbb{G}})$ is the "universal left-regular representation", $\omega \mapsto(\omega \otimes \mathrm{id})(\mathrm{W})$.

Theorem 3.2. Let $L_{*} \in C_{c b}^{l}\left(L^{1}(\mathbb{G})\right)$. There exists $x \in M\left(C_{0}^{u}(\widehat{\mathbb{G}})\right)$ with $x \lambda_{u}(\omega)=\lambda_{u}\left(L_{*}(\omega)\right)$ for $\omega \in L^{1}(\mathbb{G})$, or equivalently, $(L \otimes \mathrm{id})(\mathrm{W})=(1 \otimes x) \mathrm{W}$.

Furthermore, if $L_{*} \in \mathcal{C B}\left(L^{1}(\mathbb{G})\right)$ is any completely bounded map and $x \in M\left(C_{0}^{u}(\widehat{\mathbb{G}})\right)$ any element, such that $(L \otimes \mathrm{id})(\mathrm{W})=(1 \otimes x) \mathrm{W}$, then $L_{*}$ is a centraliser, associated with $\pi_{\widehat{\mathbb{G}}}(x) \in$ $M_{c b}^{l}\left(L^{1}(\mathbb{G})\right)$.

Proof. Again working in $L^{\infty}(\mathbb{G}) \bar{\otimes} C_{0}^{u}(\widehat{\mathbb{G}})^{\prime \prime} \subseteq \mathcal{B}\left(L^{2}(G) \otimes K\right)$, we set $X=(L \otimes \mathrm{id})(\mathrm{W}) \mathrm{W}^{*} \in$ $L^{\infty}(\mathbb{G}) \bar{\otimes} C_{0}^{u}(\widehat{\mathbb{G}})^{\prime \prime}$. By the previous lemma, we know that $X \in M\left(\mathcal{B}_{0}\left(L^{2}(\mathbb{G})\right) \otimes C_{0}^{u}(\widehat{\mathbb{G}})\right)$. We calculate that

$$
\begin{aligned}
(\Delta \otimes \mathrm{id})(X) & =(\Delta L \otimes \mathrm{id})(\mathrm{W})\left(\mathrm{W}_{13} \mathrm{~W}_{23}\right)^{*}=((L \otimes \mathrm{id}) \Delta \otimes \mathrm{id})(\mathrm{W}) \mathrm{W}_{23}^{*} \mathrm{~W}_{13}^{*} \\
& =(L \otimes \mathrm{id} \otimes \mathrm{id})\left(\mathrm{W}_{13} \mathrm{~W}_{23}\right) \mathrm{W}_{23}^{*} \mathrm{~W}_{13}^{*}=\left((L \otimes \mathrm{id})(\mathrm{W}) \mathrm{W}^{*}\right)_{13}=X_{13}
\end{aligned}
$$

By slicing on the right, and using that invariants are constant, we see that $X \in \mathbb{C} 1 \bar{\otimes} M\left(C_{0}^{u}(\widehat{\mathbb{G}})\right)$. Thus, there is $x \in M\left(C_{0}^{u}(\widehat{\mathbb{G}})\right)$ with $X=1 \otimes x$, or equivalently,

$$
(L \otimes \mathrm{id})(\mathrm{W})=(1 \otimes x) \mathrm{W} .
$$

Now suppose that all we know about $L$ is that $(L \otimes \mathrm{id})(\mathrm{W})=(1 \otimes x) \mathrm{W}$. Then

$$
\lambda_{u}\left(L_{*}(\omega)\right)=(\omega \otimes \mathrm{id})((L \otimes \mathrm{id})(\mathrm{W}))=(\omega \otimes \mathrm{id})((1 \otimes x) \mathrm{W})=x \lambda_{u}(\omega),
$$

and so, as $\lambda_{u}$ is injective, $L_{*}$ is a centraliser. As $\pi_{\widehat{\mathbb{G}}} \lambda_{u}=\lambda$, it follows that $\pi_{\widehat{\mathbb{G}}}(x) \in M_{c b}^{l}\left(L^{1}(\mathbb{G})\right)$ is the multiplier given by $L_{*}$.

Definition 3.3. Let the collection of those $x \in M\left(C_{0}^{u}(\widehat{\mathbb{G}})\right)$ associated to completely bounded left centralisers be denoted by $M_{c b}^{l, u}\left(L^{1}(\mathbb{G})\right)$.

We note that (the strict extension of) $\pi_{\widehat{\mathbb{G}}}$ restricts to a bijection $M_{c b}^{l, u}\left(L^{1}(\mathbb{G})\right) \rightarrow M_{c b}^{l}\left(L^{1}(\mathbb{G})\right)$.

While discussing multiplier algebras, we make the following remark. Let $L_{*} \in C_{c b}^{l}\left(L^{1}(\mathbb{G})\right)$. As elements of the form $(\mathrm{id} \otimes \omega)(W)$ are norm dense in $C_{0}(\mathbb{G})$, and as $(L \otimes \mathrm{id})(W)=(1 \otimes x) W$, it follows that $L$ restricts to a map $C_{0}(\mathbb{G}) \rightarrow C_{0}(\mathbb{G})$. We remark that we don't know if $L$ gives a $\operatorname{map} M\left(C_{0}(\mathbb{G})\right) \rightarrow M\left(C_{0}(\mathbb{G})\right)$. 
We next adapt an idea from the proof of [8, Theorem 4.2], which will allow us to find a "universal $C^{*}$-algebraic" version of the representation theorem of [14]. Firstly, we recall some notions from, for example, [20, Section 5.5]. For a $C^{*}$-algebra $A$ and an index set $I$, let $M C_{I}(A)$ be the families $\left(x_{i}\right)_{i \in I} \subseteq M(A)$ with $\sum_{i} x_{i}^{*} x_{i}$ strictly converging in $M(A)$. Similarly let $M R_{I}(A)$ be those $\left(x_{i}\right)$ with $\sum_{i} x_{i} x_{i}^{*}$ strictly converging, so $\left(x_{i}\right) \in M C_{I}(A)$ if and only if $\left(x_{i}^{*}\right) \in M R_{I}(A)$. For $x, y \in M C_{I}(A)$, [20, Lemma 5.28] shows that $\sum_{i} x_{i}^{*} y_{i}$ is strictly convergent and that the partial sums form a bounded family. Furthermore, these notions are stable under applying nondegenerate $*$-homomorphisms. These notions have obvious links with the (extended) Haagerup tensor product, see for example [30, Proposition 1.15].

Theorem 3.4. For $x \in M_{c b}^{l, u}\left(L^{1}(\mathbb{G})\right)$, there exists $\left(a_{i}\right),\left(b_{i}\right) \in M C_{I}\left(C_{0}^{u}(\widehat{\mathbb{G}})\right)$ with $\sum_{i}\left(1 \otimes b_{i}^{*}\right) \Delta\left(a_{i}\right)=$ $x \otimes 1$.

Proof. Let $L_{*} \in C_{c b}^{l}\left(L^{1}(\mathbb{G})\right)$ and $x$ be linked as before. Let $L(a)=S^{*}(a \otimes 1) T$ as in the proof of Lemma 3.1, and again suppose that $C_{0}^{u}(\widehat{\mathbb{G}})$ is represented on a Hilbert space $K$. Fix a unit vector $\xi \in L^{2}(\mathbb{G})$, let $\left(e_{i}\right)$ be an orthonormal basis of $L^{2}(\mathbb{G}) \otimes H$, and for each $i$, set

$$
a_{i}=\left(\mathrm{id} \otimes \omega_{\xi, e_{i}}\right)\left(\widehat{\mathbb{W}}_{13}^{*}(1 \otimes T) \widehat{\mathbb{W}}\right) .
$$

This is a slight abuse of notation; by definition, what we mean is that $a_{i} \in \mathcal{B}(K)$ is the operator

$$
\left(a_{i}(\alpha) \mid \beta\right)=\left(\widehat{\mathbb{W}}_{13}^{*}(1 \otimes T) \widehat{\mathbb{W}}(\alpha \otimes \xi) \mid \beta \otimes e_{i}\right) \quad(\alpha, \beta \in K),
$$

which makes sense as $\mathbb{W} \in M\left(C_{0}^{u}(\widehat{\mathbb{G}}) \otimes C_{0}(\mathbb{G})\right) \subseteq \mathcal{B}\left(K \otimes L^{2}(\mathbb{G})\right)$. Equivalently,

$$
\sum_{i} a_{i}(\alpha) \otimes e_{i}=\widehat{\mathbb{W}}_{13}^{*}(1 \otimes T) \widehat{\mathbb{W}}(\alpha \otimes \xi) \quad(\alpha \in K)
$$

Thus, for $\alpha, \beta \in K$,

$$
\begin{aligned}
\left(\sum_{i} a_{i}^{*} a_{i}(\alpha) \mid \beta\right) & =\left(\widehat{\mathbb{W}}_{13}^{*}(1 \otimes T) \widehat{\mathbb{W}}(\alpha \otimes \xi) \mid \widehat{W}_{13}^{*}(1 \otimes T) \widehat{\mathbb{W}}(\beta \otimes \xi)\right) \\
& =\left(\widehat{\mathbb{W}}^{*}\left(1 \otimes T^{*} T\right) \widehat{\mathbb{W}}(\alpha \otimes \xi) \mid \beta \otimes \xi\right) .
\end{aligned}
$$

So $\sum_{i} a_{i}^{*} a_{i}=\left(\mathrm{id} \otimes \omega_{\xi, \xi}\right)\left(\widehat{\mathbb{W}}^{*}\left(1 \otimes T^{*} T\right) \widehat{\mathbb{W}}\right) \in M\left(C_{0}^{u}(\widehat{\mathbb{G}})\right)$, where the sum converges weakly in $\mathcal{B}(K)$. We claim that each $a_{i} \in M\left(C_{0}^{u}(\widehat{\mathbb{G}})\right)$, and that this sum converges strictly. We shall show that for $a \in C_{0}^{u}(\widehat{\mathbb{G}})$, the sum $\sum_{i} a_{i}^{*} a_{i} a$ is norm convergent; the proof for $\sum_{i} a a_{i}^{*} a_{i}$ is similar.

Choose $\theta \in \mathcal{B}_{0}\left(L^{2}(\mathbb{G})\right)$ with $\theta(\xi)=\xi$. As $\widehat{\mathbb{W}}(a \otimes \theta) \in C_{0}^{u}(\widehat{\mathbb{G}}) \otimes \mathcal{B}_{0}\left(L^{2}(\mathbb{G})\right)$, and arguing similarly to the proof of Lemma 3.1, for $\epsilon>0$ we can find $\left(a_{j}\right)_{j=1}^{n} \subseteq C_{0}^{u}(\widehat{\mathbb{G}})$ and $\left(\xi_{j}\right)_{j=1}^{n} \subseteq L^{2}(\mathbb{G}) \otimes H$ with

$$
\left\|\widehat{\mathbb{W}}_{13}^{*}(1 \otimes T) \widehat{\mathbb{W}}(a \alpha \otimes \xi)-\sum_{j} a_{j}(\alpha) \otimes \xi_{j}\right\| \leq \epsilon\|\alpha\| \quad(\alpha \in K) .
$$

Here we used that $\widehat{\mathbb{W}}(a \alpha \otimes \xi)=\widehat{\mathbb{W}}(a \otimes \theta)(\alpha \otimes \xi)$ for any $\alpha$. For each $j$ let $\xi_{j}=\sum_{i} \xi_{j, i} e_{i}$, so equivalently we have that

$$
\left\|\sum_{i}\left(a_{i} a(\alpha)-\sum_{j} \xi_{j, i} a_{j}(\alpha)\right) \otimes e_{i}\right\| \leq \epsilon\|\alpha\| \quad(\alpha \in K) .
$$

From this (and a similar argument with $a$ on the left) it follows that indeed $a_{i} \in M\left(C_{0}^{u}(\widehat{\mathbb{G}})\right)$ for each $i$. Continuing, let $\epsilon_{0}>0$ with $\epsilon_{0} \sum_{j}\left\|a_{j}\right\| \leq \epsilon$, and choose a finite subset $I_{0} \subseteq I$ with, for each $j$

$$
\sum_{i \notin I_{0}}\left|\xi_{j, i}\right|^{2} \leq \epsilon_{0}^{2}
$$


Then

$$
\begin{aligned}
\left\|\sum_{i \notin I_{0}} a_{i} a(\alpha) \otimes e_{i}\right\| & =\left\|\sum_{i} a_{i} a(\alpha) \otimes e_{i}-\sum_{i \in I_{0}} a_{i} a(\alpha) \otimes e_{i}\right\| \\
& \leq \epsilon\|\alpha\|+\left\|\sum_{i} \sum_{j} \xi_{j, i} a_{j}(\alpha) \otimes e_{i}-\sum_{i \in I_{0}} a_{i} a(\alpha) \otimes e_{i}\right\| \\
& \leq \epsilon\|\alpha\|+\left\|\sum_{i \notin I_{0}} \sum_{j} \xi_{j, i} a_{j}(\alpha) \otimes e_{i}\right\|+\left\|\sum_{i \in I_{0}}\left(\sum_{j} \xi_{j, i} a_{j}(\alpha)-a_{i} a(\alpha)\right) \otimes e_{i}\right\| \\
& \leq \epsilon\|\alpha\|+\|\alpha\| \epsilon_{0} \sum_{j}\left\|a_{j}\right\|+\epsilon\|\alpha\| \leq 3 \epsilon\|\alpha\| .
\end{aligned}
$$

Finally, we then see that for $\alpha, \beta \in K$,

$$
\begin{aligned}
\mid\left(\sum_{i \in I_{0}} a_{i}^{*} a_{i} a(\alpha) \mid \beta\right) & -\left(\widehat{\mathbb{W}}^{*}\left(1 \otimes T^{*} T\right) \widehat{\mathbb{W}}(a(\alpha) \otimes \xi) \mid \beta \otimes \xi\right) \mid \\
& =\left|\left(\sum_{i \in I_{0}} a_{i} a(\alpha) \otimes e_{i} \mid \sum_{j} a_{j}(\beta) \otimes e_{j}\right)-\left(\sum_{i} a_{i} a(\alpha) \otimes e_{i} \mid \sum_{j} a_{j}(\beta) \otimes e_{j}\right)\right| \\
& \leq\|T\|\|\beta\|\left\|\sum_{i \notin I_{0}} a_{i} a(\alpha) \otimes e_{i}\right\| .
\end{aligned}
$$

Putting these together, we see that

$$
\left\|\sum_{i \in I_{0}} a_{i}^{*} a_{i} a-\left(\mathrm{id} \otimes \omega_{\xi, \xi}\right)\left(\widehat{\mathbb{W}}^{*}\left(1 \otimes T^{*} T\right) \widehat{\mathbb{W}}\right) a\right\| \leq 3 \epsilon
$$

as required. Thus $\left(a_{i}\right) \in M C_{I}\left(C_{0}^{u}(\widehat{\mathbb{G}})\right)$.

We similarly define $b_{i}=\left(\mathrm{id} \otimes \omega_{\xi, e_{i}}\right)\left(\widehat{\mathbb{W}}_{13}^{*}(1 \otimes S) \widehat{W}\right)$. Now, using a similar abuse of notation,

$$
\Delta\left(a_{i}\right)=\left(\mathrm{id} \otimes \mathrm{id} \otimes \omega_{\xi, e_{i}}\right)\left(\widehat{\mathbb{W}}_{24}^{*} \widehat{\mathbb{W}}_{14}^{*}(1 \otimes 1 \otimes T) \widehat{\mathbb{W}}_{13} \widehat{\mathbb{W}}_{23}\right)
$$

Then

$$
\begin{aligned}
& \sum_{i}\left(1 \otimes b_{i}\right)^{*} \Delta\left(a_{i}\right)=\sum_{i}\left(\mathrm{id} \otimes \mathrm{id} \otimes \omega_{e_{i}, \xi}\right)\left(\widehat{\mathbb{W}}_{23}^{*}\left(1 \otimes 1 \otimes S^{*}\right) \widehat{\mathbb{W}}_{24}\right) \\
& \left(\mathrm{id} \otimes \mathrm{id} \otimes \omega_{\xi, e_{i}}\right)\left(\widehat{\mathrm{W}}_{24}^{*} \widehat{\mathbb{W}}_{14}^{*}(1 \otimes 1 \otimes T) \widehat{\mathbb{W}}_{13} \widehat{\mathrm{W}}_{23}\right) \\
& =\left(\mathrm{id} \otimes \mathrm{id} \otimes \omega_{\xi, \xi}\right)\left(\widehat{\mathrm{W}}_{23}^{*}\left(1 \otimes 1 \otimes S^{*}\right) \widehat{\mathrm{W}}_{24} \widehat{\mathrm{W}}_{24}^{*} \widehat{\mathrm{W}}_{14}^{*}(1 \otimes 1 \otimes T) \widehat{\mathrm{W}}_{13} \widehat{\mathrm{W}}_{23}\right) \\
& =\left(\mathrm{id} \otimes \mathrm{id} \otimes \omega_{\xi, \xi}\right)\left(\widehat{\mathrm{W}}_{23}^{*}\left(1 \otimes 1 \otimes S^{*}\right) \widehat{\mathrm{W}}_{14}^{*}(1 \otimes 1 \otimes T) \widehat{\mathrm{W}}_{13} \widehat{\mathrm{W}}_{23}\right) \\
& =\left(\mathrm{id} \otimes \mathrm{id} \otimes \omega_{\xi, \xi}\right)\left(\widehat{\mathrm{W}}_{23}^{*}(\mathrm{id} \otimes L)\left(\widehat{\mathrm{W}}^{*}\right)_{13} \widehat{\mathrm{W}}_{13} \widehat{\mathrm{W}}_{23}\right) \text {. }
\end{aligned}
$$

As $\widehat{\mathbb{W}}^{*}=\sigma(\mathrm{W})$ and $(L \otimes \mathrm{id})(\mathrm{W})=(1 \otimes x) \mathrm{W}$, it follows that

$$
\sum_{i}\left(1 \otimes b_{i}\right)^{*} \Delta\left(a_{i}\right)=\left(\mathrm{id} \otimes \mathrm{id} \otimes \omega_{\xi, \xi}\right)\left(\widehat{\mathbb{W}}_{23}^{*}(x \otimes 1)_{13}\left(\widehat{\mathbb{W}}^{*}\right)_{13} \widehat{\mathbb{W}}_{13} \widehat{\mathbb{W}}_{23}\right)=x \otimes 1,
$$

as claimed.

We now state a converse to the previous result, and show how to recover the original centraliser. 
Theorem 3.5. Let $\left(a_{i}\right),\left(b_{i}\right) \in M C_{I}\left(C_{0}^{u}(\widehat{\mathbb{G}})\right)$ be such that $\sum_{i}\left(1 \otimes b_{i}^{*}\right) \Delta\left(a_{i}\right)=x \otimes 1$ for some $x \in M\left(C_{0}^{u}(\widehat{\mathbb{G}})\right)$. Then $x \in M_{c b}^{l, u}\left(L^{1}(\mathbb{G})\right)$ and the associated $L_{*} \in C_{c b}^{l}\left(L^{1}(\mathbb{G})\right)$ is given by $L(a)=$ $\sum_{i} \pi_{\widehat{\mathbb{G}}}\left(b_{i}\right)^{*} a \pi_{\widehat{\mathbb{G}}}\left(a_{i}\right)$ for $a \in L^{\infty}(\mathbb{G})$, with convergence weakly in $\mathcal{B}\left(L^{2}(\mathbb{G})\right)$.

Proof. Define $L: L^{\infty}(\mathbb{G}) \rightarrow \mathcal{B}\left(L^{2}(\mathbb{G})\right) ; a \mapsto \sum_{i} \pi_{\widehat{\mathbb{G}}}\left(b_{i}\right)^{*} a \pi_{\widehat{\mathbb{G}}}\left(a_{i}\right)$, so that $L$ is a normal completely bounded map. Consider then

$$
\begin{aligned}
(L \otimes \mathrm{id})(\mathrm{W}) & =\sigma \sum_{i}\left(1 \otimes \pi_{\widehat{\mathbb{G}}}\left(b_{i}\right)^{*}\right) \widehat{\mathbb{W}}^{*}\left(1 \otimes \pi_{\widehat{\mathbb{G}}}\left(a_{i}\right)\right) \\
& =\sigma \sum_{i}\left(1 \otimes \pi_{\widehat{\mathbb{G}}}\left(b_{i}\right)^{*}\right) \widehat{\mathbb{W}}^{*}\left(1 \otimes \pi_{\widehat{\mathbb{G}}}\left(a_{i}\right)\right) \widehat{\mathbb{W} \widehat{W}^{*}} \\
& =\sigma \sum_{i}\left(1 \otimes \pi_{\widehat{\mathbb{G}}}\left(b_{i}\right)^{*}\right)\left(\mathrm{id} \otimes \pi_{\widehat{\mathbb{G}}}\right) \Delta\left(a_{i}\right) \widehat{\mathbb{W}}^{*} \\
& =\sigma\left(\left(\mathrm{id} \otimes \pi_{\widehat{\mathbb{G}}}\right)\left(\sum_{i}\left(1 \otimes b_{i}^{*}\right) \Delta\left(a_{i}\right)\right) \widehat{\mathbb{W}}^{*}\right) \\
& =(1 \otimes x) \mathrm{W} .
\end{aligned}
$$

By applying id $\otimes \pi_{\widehat{G}}$ we also see that $(L \otimes \mathrm{id})(W)=\left(1 \otimes \pi_{\widehat{\mathbb{G}}}(x)\right)(W)$. As slices (id $\left.\otimes \widehat{\omega}\right)(W)$, with $\widehat{\omega} \in L^{1}(\widehat{\mathbb{G}})$, form a weak ${ }^{*}$-dense subspace of $L^{\infty}(\mathbb{G})$, this calculation shows that $L$ does indeed map $L^{\infty}(\mathbb{G})$ to $L^{\infty}(\mathbb{G})$. Furthermore, we have now verified the condition in Theorem 3.2 and so $L_{*}$ is a left completely bounded centraliser, associated to the "universal" multiplier $x$, which completes the proof.

\subsection{Multipliers and morphisms}

Throughout this section, let $\mathbb{G}, \mathbb{H}$ be locally compact quantum groups, and let $\mathbb{G} \rightarrow \mathbb{H}$ be a morphism, represented by $\phi: C_{0}^{u}(\mathbb{H}) \rightarrow M\left(C_{0}^{u}(\mathbb{G})\right), U \in M\left(C_{0}(\mathbb{G}) \otimes C_{0}(\widehat{\mathbb{H}})\right)$ and $\beta: C_{0}(\mathbb{H}) \rightarrow$ $M\left(C_{0}(\mathbb{G}) \otimes C_{0}(\mathbb{H})\right)$. Let $\widehat{\phi}: C_{0}^{u}(\widehat{\mathbb{G}}) \rightarrow M\left(C_{0}^{u}(\widehat{\mathbb{H}})\right)$ be the dual Hopf $*$-homomorphism.

The following is then the most natural way that morphisms and multipliers could interact in. We shall then go on to show how the other "pictures" also interact in natural ways.

Theorem 3.6. The map $\widehat{\phi}$ restricts to a homomorphism $M_{c b}^{l, u}\left(L^{1}(\mathbb{G})\right) \rightarrow M_{c b}^{l, u}\left(L^{1}(\mathbb{H})\right)$.

Proof. Let $L_{*} \in C_{c b}^{l}\left(L^{1}(\mathbb{G})\right)$ and let $\left(a_{i}\right),\left(b_{i}\right) \in M C_{I}\left(C_{0}^{u}(\widehat{\mathbb{G}})\right)$ as in Theorem 3.4, so $L_{*}$ is associated to $x \in M_{c b}^{l, u}\left(L^{1}(\mathbb{G})\right)$ where $\sum_{i}\left(1 \otimes b_{i}^{*}\right) \Delta\left(a_{i}\right)=x \otimes 1$.

As $\widehat{\phi}$ is a Hopf $*$-homomorphism,

$$
\sum_{i}\left(1 \otimes \widehat{\phi}\left(b_{i}^{*}\right)\right) \Delta\left(\widehat{\phi}\left(a_{i}\right)\right)=(\widehat{\phi} \otimes \widehat{\phi}) \sum_{i}\left(1 \otimes b_{i}^{*}\right) \Delta\left(a_{i}\right)=\widehat{\phi}(x) \otimes 1
$$

and so an application of Theorem 3.5 shows that there is $L^{\prime} \in C_{c b}^{l}\left(L^{1}(\mathbb{H})\right)$ associated with $\widehat{\phi}(x) \in$ $M_{c b}^{l, u}\left(L^{1}(\mathbb{H})\right)$ as required.

Remark 3.7. The "classical" situation here is detailed in [27, Section 6.1], where it is shown that a group homomorphism $G \rightarrow H$, which induces a Hopf $*$-homomorphism $C_{0}(H) \rightarrow C_{b}(G)$, restricts to a map $M_{c b} A(H) \rightarrow M_{c b} A(G)$. In our language, we would start with a morphism $\widehat{H} \rightarrow \widehat{G}$, say given by $\phi: C^{*}(G) \rightarrow M\left(C^{*}(H)\right)$, and then consider the dual $\widehat{\phi}: C_{0}(H) \rightarrow C_{b}(G)$. Hence we exactly recover the classical result, once we have the "duality convention" correct. 
One way to find centralisers of $L^{1}(\mathbb{G})$ is to embed $L^{1}(\mathbb{G})$ into $C_{0}^{u}(\mathbb{G})^{*}$, where it becomes a closed two-sided ideal, and so (left) multiplication by elements of $C_{0}^{u}(\mathbb{G})^{*}$ define members of $C_{c b}^{l}\left(L^{1}(\mathbb{G})\right)$ (and all completely positive centralisers arise in this way, [7]). The following shows that morphisms, from the Hopf $*$-homomorphism perspective, behave as expected.

Proposition 3.8. We have the commutative diagram

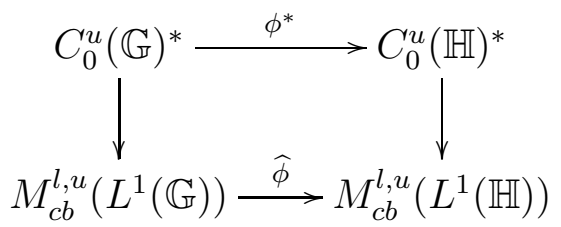

where the bottom arrow is given by the previous theorem.

Proof. Let $\mu \in C_{0}^{u}(\mathbb{G})^{*}$ and write $\pi_{\mathbb{G}}^{*}: L^{1}(\mathbb{G}) \rightarrow C_{0}^{u}(\mathbb{G})^{*}$ for the embedding, which is a completely isometric algebra homomorphism. Let $\mu$ induce $L_{*} \in C_{c b}^{l}\left(L^{1}(\mathbb{G})\right)$, which means that $\pi_{\mathbb{G}}^{*}\left(L_{*}(\omega)\right)=$ $\mu \pi_{\mathbb{G}}^{*}(\omega)=(\mu \otimes \omega)\left(\right.$ id $\left.\otimes \pi_{\mathbb{G}}\right) \Delta$ for each $\omega \in L^{1}(\mathbb{G})$. Then

$$
\begin{aligned}
(L \otimes \mathrm{id})(\mathrm{W}) & =\left(L \pi_{\mathbb{G}} \otimes \mathrm{id}\right)(\mathbb{W})=\left(\left(\mu \otimes \pi_{\mathbb{G}}\right) \Delta \otimes \mathrm{id}\right)(\mathbb{W})=\left(\mu \otimes \pi_{\mathbb{G}} \otimes \mathrm{id}\right)\left(\mathbb{W}_{13} \mathbb{W}_{23}\right) \\
& =((\mu \otimes \mathrm{id})(\mathbb{W}) \otimes 1) \mathrm{W} .
\end{aligned}
$$

By Theorem $\left[3.2\right.$, we see that $x=(\mu \otimes \mathrm{id})(\mathbb{W}) \in M\left(C_{0}^{u}(\widehat{\mathbb{G}})\right) \in M_{c b}^{l, u}\left(L^{1}(\mathbb{G})\right)$ is associated to $L$ and hence to $\mu$.

Similarly let $\phi^{*}(\mu) \in C_{0}^{u}(\mathbb{H})^{*}$ induce $L_{*}^{\prime} \in C_{c b}^{l}\left(L^{1}(\mathbb{H})\right)$ which is thus associated to $x^{\prime} \in M_{c b}^{l, u}\left(L^{1}(\mathbb{H})\right)$ where

$$
x^{\prime}=\left(\phi^{*}(\mu) \otimes \mathrm{id}\right)\left(\mathbb{W}_{\mathbb{H}}\right)=(\mu \otimes \mathrm{id})\left((\phi \otimes \mathrm{id})\left(\mathbb{W}_{\mathbb{H}}\right)\right)=(\mu \otimes \mathrm{id})\left((\mathrm{id} \otimes \widehat{\phi})\left(\mathbb{W}_{\mathbb{G}}\right)\right)=\widehat{\phi}(x)
$$

as required to show that the diagram commutes.

We now demonstrate a similar link at the level of centralisers, and not multipliers, using bicharacters and quantum group homomorphisms (a picture not really explored in [27], for example, but see Remark 3.12 below for links with [15]).

Lemma 3.9. Let $L_{*} \in C_{c b}^{l}\left(L^{1}(\mathbb{G})\right)$ and $x \in M_{c b}^{l, u}\left(L^{1}(\mathbb{G})\right)$ be linked, and let $U$ be the bicharacter representing the morphism $\mathbb{G} \rightarrow \mathbb{H}$. Then we have that $(L \otimes \mathrm{id})(U)=\left(1 \otimes \pi_{\widehat{\mathbb{H}}} \widehat{\phi}(x)\right) U$.

Proof. As $U=\left(\pi_{\mathbb{G}} \phi \otimes \mathrm{id}\right)\left(\mathbb{W}_{\mathbb{H}}\right)$ we see that

$$
\begin{aligned}
(L \otimes \mathrm{id})(U) & =\left(L \pi_{\mathbb{G}} \phi \otimes \pi_{\widehat{\mathbb{H}}}\right)\left(\mathbb{W}_{\mathbb{H}}\right)=\left(L \pi_{\mathbb{G}} \otimes \pi_{\widehat{\mathbb{H}}} \widehat{\phi}\right)\left(\mathbb{W}_{\mathbb{G}}\right)=\left(L \otimes \pi_{\widehat{\mathbb{H}}} \widehat{\phi}\right)\left(\mathrm{W}_{\mathbb{G}}\right) \\
& =\left(\mathrm{id} \otimes \pi_{\widehat{\mathbb{H}}} \widehat{\phi}\right)\left((1 \otimes x) \mathrm{W}_{\mathbb{G}}\right)=\left(1 \otimes \pi_{\widehat{\mathbb{H}}} \widehat{\phi}(x)\right)\left(\mathrm{id} \otimes \pi_{\widehat{\mathbb{H}}} \widehat{\phi}\right)\left(\mathrm{W}_{\mathbb{G}}\right) \\
& =\left(1 \otimes \pi_{\widehat{\mathbb{H}}} \widehat{\phi}(x)\right) U,
\end{aligned}
$$

as claimed.

Proposition 3.10. Let $L_{*} \in C_{c b}^{l}\left(L^{1}(\mathbb{G})\right)$ be mapped to $L_{*}^{\prime} \in C_{c b}^{l}\left(L^{1}(\mathbb{H})\right)$ by the morphism $\mathbb{G} \rightarrow \mathbb{H}$, and let $U$ be the bicharacter representing this morphism. Then

$$
1 \otimes L^{\prime}(a)=U(L \otimes \mathrm{id})\left(U^{*}(1 \otimes a) U\right) U^{*} \quad\left(a \in L^{\infty}(\mathbb{H}) .\right.
$$


Proof. By weak ${ }^{*}$-continuity, it is enough to show this for $a=(\mathrm{id} \otimes \omega)\left(W_{\mathbb{H}}\right)$. With this in mind, the claim is equivalent to

$$
1 \otimes\left(L^{\prime} \otimes \mathrm{id}\right)\left(W_{\mathbb{H}}\right)=U_{12}(L \otimes \mathrm{id} \otimes \mathrm{id})\left(U_{12}^{*} W_{\mathbb{H}, 23} U_{12}\right) U_{12}^{*}
$$

Now, we have that $W_{\mathbb{H}, 23} U_{12} W_{\mathbb{H}, 23}^{*}=U_{12} U_{13}$ as $U$ is a bicharacter, and so the right hand side equals, using the previous lemma,

$$
\begin{aligned}
U_{12}(L \otimes \mathrm{id} \otimes \mathrm{id})\left(U_{13} W_{\mathbb{H}, 23}\right) U_{12}^{*} & =U_{12}\left(\left(1 \otimes \pi_{\widehat{\mathbb{H}}} \widehat{\phi}(x)\right) U\right)_{13} W_{\mathbb{H}, 23} U_{12}^{*} \\
& =U_{12}\left(1 \otimes 1 \otimes \pi_{\widehat{\mathbb{H}}} \widehat{\phi}(x)\right) U_{13} W_{\mathbb{H}, 23} U_{12}^{*} \\
& =U_{12}\left(1 \otimes 1 \otimes \pi_{\widehat{\mathbb{H}}} \widehat{\phi}(x)\right) U_{12}^{*} W_{\mathbb{H}, 23} \\
& =1 \otimes\left(1 \otimes \pi_{\widehat{\mathbb{H}}} \widehat{\phi}(x)\right)\left(W_{\mathbb{H}}\right)=1 \otimes\left(L^{\prime} \otimes \mathrm{id}\right)\left(W_{\mathbb{H}}\right)
\end{aligned}
$$

as claimed.

Recalling that the quantum group homomorphism $\beta$ satisfies that $\beta(a)=U^{*}(1 \otimes a) U$ for $a \in L^{\infty}(\mathbb{H})$, the following is immediate. Notice that we can think of this as being a generalisation of the covariance condition which defines what it means for $L$ to be (the adjoint of) a centraliser.

Corollary 3.11. Let $L_{*} \in C_{c b}^{l}\left(L^{1}(\mathbb{G})\right)$ be mapped to $L_{*}^{\prime} \in C_{c b}^{l}\left(L^{1}(\mathbb{H})\right)$ by our morphism, which is represented by the quantum group homomorphism $\beta$. Then $\beta L^{\prime}=(L \otimes \mathrm{id}) \beta$.

This makes immediate sense if we work at the von Neumann algebra level, and regard $\beta$ as a map $L^{\infty}(\mathbb{G}) \rightarrow L^{\infty}(\mathbb{G}) \bar{\otimes} L^{\infty}(\mathbb{H})$. It is not clear how to, a priori, give a purely $C^{*}$-algebraic interpretation of this.

Remark 3.12. It is possible to work purely at the level of bicharacters and centralisers, without passing to multipliers and Hopf $*$-homomorphisms. Indeed, let $U \in L^{\infty}(\mathbb{G}) \bar{\otimes} L^{\infty}(\widehat{\mathbb{H}})$ represent $\mathbb{G} \rightarrow \mathbb{H}$, and let $L_{*} \in C_{c b}^{l}(\mathbb{G})$. Then consider $(L \otimes \mathrm{id})(U) U^{*}$. By applying $(\Delta \otimes \mathrm{id})$, and arguing as in the proof of Theorem 3.2 , we find $x \in L^{\infty}(\widehat{\mathbb{H}})$ with $(L \otimes \mathrm{id})(U)=(1 \otimes x) U$. Of course, $x$ will turn out to be the multiplier associated to $L^{\prime}$.

For $a \in L^{\infty}(\mathbb{H})$, we can now consider $U(L \otimes \mathrm{id})\left(U^{*}(1 \otimes a) U\right) U^{*}$. The proof of Proposition 3.10 still works, and we find that if $a=(\mathrm{id} \otimes \omega)\left(W_{\mathbb{H}}\right)$ then there is $L^{\prime}(a) \in L^{\infty}(\mathbb{H})$ with $U^{*}\left(1 \otimes L^{\prime}(a)\right) U=$ $(L \otimes \mathrm{id})\left(U^{*}(1 \otimes a) U\right)$. Indeed, $L^{\prime}(a)=(\mathrm{id} \otimes \omega x)\left(W_{\mathbb{H}}\right)$. By normality, it follows easily that $L^{\prime}$ extends to a completely bounded normal map $L^{\infty}(\mathbb{H}) \rightarrow L^{\infty}(\mathbb{H})$, and also $\left(L^{\prime} \otimes \mathrm{id}\right)\left(W_{\mathbb{H}}\right)=(1 \otimes x) W_{\mathbb{H}}$ and so $L^{\prime}$ is the adjoint of a centraliser.

This argument, and Proposition 3.10, should also be compared with [15, Theorem 2.1], where the relation between centralisers and actions of quantum groups (at the von Neumann algebra level) is explored: we can apply this to $\beta$, as $\beta$ is a (special sort of) coaction. Notice that our use of the "invariants are constant" approach allows us to avoid weight theory, and the use of crossed product theory.

Let us finally make some remarks about operator space structures. The space $C_{c b}^{l}\left(L^{1}(\mathbb{G})\right)$ inherits a natural operator space structure as a subspace of $\mathcal{C B}\left(L^{1}(\mathbb{G})\right) \subseteq \mathcal{C B}\left(L^{\infty}(\mathbb{G})\right)$, and using this, we induce an operator space structure on $M_{c b}^{l}\left(L^{1}(\mathbb{G})\right)$ and $M_{c b}^{l, u}\left(L^{1}(\mathbb{G})\right)$. As $\beta$ is a complete isometry, it follows more or less immediately from Corollary 3.11 that the map $L \mapsto L^{\prime}$ is a complete contraction. Let us formally state this.

Theorem 3.13. A morphism $\mathbb{G} \rightarrow \mathbb{H}$ induces a complete contraction $C_{c b}^{l}\left(L^{1}(\mathbb{G})\right) \rightarrow C_{c b}^{l}\left(L^{1}(\mathbb{H})\right)$ and thus a complete contraction $M_{c b}^{l, u}\left(L^{1}(\mathbb{G})\right) \rightarrow M_{c b}^{l, u}\left(L^{1}(\mathbb{H})\right)$. 


\subsection{The representation theorem}

Let $\mathcal{C B}_{L^{\infty}(\widehat{\mathbb{G}})^{\prime}}^{\sigma, L^{\infty}(\mathbb{B})}\left(\mathcal{B}\left(L^{2}(\mathbb{G})\right)\right)$ be the space of normal completely bounded maps $\Phi: \mathcal{B}\left(L^{2}(\mathbb{G})\right) \rightarrow$ $\mathcal{B}\left(L^{2}(\mathbb{G})\right)$ which restrict to maps $L^{\infty}(\mathbb{G}) \rightarrow L^{\infty}(\mathbb{G})$, and which are $L^{\infty}(\widehat{\mathbb{G}})^{\prime}$-bimodule maps. The paper [14] shows that $M_{c b}^{l}\left(L^{1}(\mathbb{G})\right)$ is (completely isometrically) isomorphic to this space.

Given $L_{*} \in C_{c b}^{l}\left(L^{\infty}(\mathbb{G})\right)$ we can extend $L$ to all of $\mathcal{B}\left(L^{2}(\mathbb{G})\right)$ in such a way that $L$ becomes a $L^{\infty}(\widehat{\mathbb{G}})^{\prime}$-bimodule map. Indeed, we claim that for each $x \in \mathcal{B}\left(L^{2}(\mathbb{G})\right)$ there is $\Phi(x) \in \mathcal{B}\left(L^{2}(\mathbb{G})\right)$ with

$$
1 \otimes \Phi(x)=W\left((L \otimes \mathrm{id})\left(W^{*}(1 \otimes x) W\right)\right) W^{*} .
$$

Then $\Phi$ is easily seen to be completely bounded, normal, to extend $L$, and to be a $L^{\infty}(\widehat{\mathbb{G}})^{\prime}$ bimodule map, as $W \in L^{\infty}(\mathbb{G}) \bar{\otimes} L^{\infty}(\widehat{\mathbb{G}})$. That $\Phi$ exists can shown using the "invariants are constant" technique, see [7, Proposition 3.2]. Here we shall follow the original approach of [14], and use that the linear span of $\left\{a b: a \in L^{\infty}(\mathbb{G}), b \in L^{\infty}(\widehat{\mathbb{G}})^{\prime}\right\}$ is weak ${ }^{*}$-dense in $\mathcal{B}\left(L^{2}(\mathbb{G})\right)$, see for example [7, Theorem 2.2]. For such $a b$ we see that

$$
W\left((L \otimes \mathrm{id})\left(W^{*}(1 \otimes a b) W\right)\right) W^{*}=W((L \otimes \mathrm{id}) \Delta(a)) W^{*}(1 \otimes b)=1 \otimes L(a) b .
$$

Thus $\Phi$ exists, $\Phi(a b)=L(a) b$ and similarly $\Phi(b a)=b L(a)$, so establishing all the needed properties. That any $\Phi \in \mathcal{C B}_{L^{\infty}(\widehat{\mathbb{G}})^{\prime}}^{\sigma, L^{\infty}(\mathbb{B})}\left(\mathcal{B}\left(L^{2}(\mathbb{G})\right)\right)$ arises in exactly this way is more intricate, see [14, Theorem 4.10].

Proposition 3.14. Continuing with this notation, let $L_{*} \in C_{c b}^{l}\left(L^{1}(\mathbb{G})\right)$ be extended to $\Phi$, and using a morphism $\mathbb{G} \rightarrow \mathbb{H}$, let $L_{*}$ be mapped to $L_{*}^{\prime} \in C_{c b}^{l}\left(L^{1}(\mathbb{H})\right)$, which is extended to $\Phi^{\prime}$. Then $U^{*}\left(1 \otimes \Phi^{\prime}(x)\right) U=(\Phi \otimes \mathrm{id})\left(U^{*}(1 \otimes x) U\right)$ for all $x \in \mathcal{B}\left(L^{2}(\mathbb{H})\right)$ where again $U$ is the bicharacter associated to our morphism.

Proof. By weak*-continuity, it suffices to verify this for $x=a b$ with $a \in L^{\infty}(\mathbb{H})$ and $b \in L^{\infty}(\widehat{\mathbb{H}})^{\prime}$. However, as $U \in L^{\infty}(\mathbb{G}) \bar{\otimes} L^{\infty}(\widehat{\mathbb{H}})$, we see that $U^{*}(1 \otimes x) U \in L^{\infty}(\mathbb{G}) \bar{\otimes} \mathcal{B}\left(L^{2}(\mathbb{H})\right)$ and so

$$
\begin{aligned}
(\Phi \otimes \mathrm{id})\left(U^{*}(1 \otimes x) U\right) & =(L \otimes \mathrm{id})\left(U^{*}(1 \otimes a) U\right)(1 \otimes b)=U^{*}\left(1 \otimes L^{\prime}(a)\right) U(1 \otimes b) \\
& =U^{*}\left(1 \otimes L^{\prime}(a) b\right) U=U^{*}\left(1 \otimes \Phi^{\prime}(x)\right) U,
\end{aligned}
$$

using Proposition 3.10 and the discussion above.

\section{Intrinsic groups}

The intrinsic group of a Kac algebra was stuided by De Canniére in [5] (for example), and for locally compact quantum groups by Kalantar and Neufang in [16]. In this section, we will show that the assignment of a locally compact quantum group to its intrinsic group is a functor between the appropriate categories, show that we can identify the intrinsic group as the "maximal" classical subgroup, and then use this to show that the "intrinsic functor" is the left adjoint to the inclusion functor from locally compact groups to locally compact quantum groups. In fact, we shall show that the intrinsic group is a closed subgroup, in the sense of [10], in fact, in the strong Vaes closed sense.

There are a number of different, equivalent, definitions of the intrinsic group, and these different definitions have interesting interactions with the different presentations of a morphism between quantum groups. We wish to be rather careful about the isomorphisms involved, and furthermore, we also want to consider the interaction with $C_{0}^{u}(\mathbb{G})$. Thus, we shall expound some of the results from [16] in detail. 
The following is the key technical lemma; two rather different proofs can be found in [9, Theorem 3.2] and [16, Theorem 3.9].

Proposition 4.1. Let $\mathbb{G}$ be a locally compact quantum group, and let $x \in L^{\infty}(\mathbb{G})$ be non-zero with $\Delta(x)=x \otimes x$. Then $x$ is unitary, and $x \in M\left(C_{0}(\mathbb{G})\right)$.

That is, all characters on $L^{1}(\mathbb{G})$ arise from one-dimensional unitary corepresentations of $\mathbb{G}$.

Lemma 4.2. Let $x \in M\left(C_{0}^{u}(\mathbb{G})\right)$ with $\Delta(x)=x \otimes x$. Then $x$ is unitary.

Proof. Let $y=\pi_{\mathbb{G}}(x) \in M\left(C_{0}(\mathbb{G})\right)$ so $\Delta(y)=y \otimes y$ and hence $y$ is unitary. Then we use that

$$
x \otimes y=\left(\mathrm{id} \otimes \pi_{\mathbb{G}}\right) \Delta(x)=\mathbb{W}^{*}(1 \otimes y) \mathbb{W}
$$

and so $x \otimes 1=\left(1 \otimes y^{*}\right) \mathbb{W}^{*}(1 \otimes y) \mathbb{W} \in M\left(C_{0}^{u}(\mathbb{G}) \otimes \mathcal{B}_{0}\left(L^{2}(\mathbb{G})\right)\right)$ is unitary, and so $x$ is unitary.

The following then states the different, equivalent, definitions of the intrinsic group, compare [16. Theorem 3.12]. We claim that the following sets, given the stated topologies, are locally compact groups, and are all homeomorphic (for maps to be defined shortly):

1. The collection of completely positive, completely isometric isomorphisms in $C_{c b}^{l}\left(L^{1}(\mathbb{G})\right)$, with composition as the group product, and the strong operator topology, denoted by $\tilde{\mathbb{G}}$;

2. The spectrum of the $C^{*}$-algebra $C_{0}^{u}(\mathbb{G})$, that is, the collection of non-zero characters on $C_{0}^{u}(\mathbb{G})$ with the relative weak*-topology, and the product induced by $\Delta$, denoted by $\operatorname{sp}\left(C_{0}^{u}(\mathbb{G})\right)$;

3. The intrinsic group of $L^{\infty}(\widehat{\mathbb{G}})$, namely $\operatorname{Gr}(\widehat{\mathbb{G}})=\left\{\widehat{u} \in L^{\infty}(\mathbb{G}): \widehat{\Delta}(\widehat{u})=\widehat{u} \otimes \widehat{u}, \widehat{u} \neq 0\right\}$, with the product from $L^{\infty}(\widehat{\mathbb{G}})$, and the relative weak*-topology;

4. The "universal intrinsic group" of $C_{0}^{u}(\mathbb{G})$, namely $\operatorname{Gr}_{u}(\widehat{\mathbb{G}})=\left\{\widehat{u} \in M\left(C_{0}^{u}(\widehat{\mathbb{G}})\right): \widehat{\Delta}(\widehat{u})=\right.$ $\widehat{u} \otimes \widehat{u}, \widehat{u} \neq 0\}$ with the product from $M\left(C_{0}^{u}(\widehat{\mathbb{G}})\right)$, and the relative strict topology.

Let us note that the "intrinsic group" is often defined by requiring that $\widehat{u}$ be invertible; but by our technical lemma, $\widehat{u}$ is automatically unitary. We note that (44) is a new equivalence not previously studied.

We now define the maps between these sets. We choose slightly different conventions to [16], in particular, swap $\widehat{u}$ for $\widehat{u}^{*}$, as our conventions seem more natural given the later results. Given $\widehat{u} \in \operatorname{Gr}(\widehat{\mathbb{G}})$, we identify $M\left(C_{0}(\widehat{\mathbb{G}})\right)$ with $M\left(\mathbb{C} \otimes C_{0}(\widehat{\mathbb{G}})\right)$ and then observe that $(\mathrm{id} \otimes \widehat{\Delta})(\widehat{u})=\widehat{u}_{13} \widehat{u}_{12}$. So by $\left[17\right.$, Proposition 5.3] there is a non-degenerate $*$-homomorphism $\gamma: C_{0}^{u}(\mathbb{G}) \rightarrow \mathbb{C}$, that is, $\gamma \in \operatorname{sp}\left(C_{0}^{u}(\mathbb{G})\right)$, with $u=(\gamma \otimes \mathrm{id})(\mathbb{W})$. It is easy to see that this in fact gives a bijection between $\operatorname{sp}\left(C_{0}^{u}(\mathbb{G})\right)$ and $\operatorname{Gr}(\widehat{\mathbb{G}})$.

By pushing things to the universal level, and using $\mathbb{W}$ and (the dual version of) [17, Proposition 6.5], we also get a bijection between $\operatorname{sp}\left(C_{0}^{u}(\mathbb{G})\right)$ and $\operatorname{Gr}_{u}(\widehat{\mathbb{G}})$ which identifies $\gamma$ with $(\gamma \otimes$ id $)(\mathbb{W})$. Then the strict extension of $\pi: C_{0}^{u}(\mathbb{G}) \rightarrow C_{0}(\mathbb{G})$ restricts to a bijection between $\operatorname{Gr}(\widehat{\mathbb{G}})$ and $\mathrm{Gr}_{u}(\widehat{\mathbb{G}})$.

Finally, the bijection between $\tilde{\mathbb{G}}$ and $\operatorname{Gr}(\widehat{\mathbb{G}})$ follows from [14, Theorem 4.7], compare [16, Theorem 3.7]. In the remainder of this section, we shall give an alternative proof, using [7], and also give a concise proof that the maps constructed are homeomorphisms; of course, these results are new for $\operatorname{Gr}_{u}(\widehat{\mathbb{G}})$.

In the next section, we start to study how morphisms and intrinsic groups interact. The following, which is really not made explicit in [16], will be vital for that purpose.

Theorem 4.3. The bijection between $C_{c b}^{l}\left(L^{1}(\mathbb{G})\right)$ and $M_{c b}^{l}\left(L^{1}(\mathbb{G})\right) \subseteq M\left(C_{0}(\widehat{\mathbb{G}})\right)$ restricts to a bijection between $\tilde{\mathbb{G}}$ and $\operatorname{Gr}(\widehat{\mathbb{G}})$. Furthermore, if $L_{*}$ and $\widehat{u}$ are thus associated, then $L(x)=\widehat{u}^{*} x \widehat{u}$ for $x \in L^{\infty}(\mathbb{G})$. 
Proof. We use the main result of [7], which tells us that there is a natural bijection between completely positive multipliers of $L^{1}(\mathbb{G})$ and $C_{0}^{u}(\mathbb{G})_{+}^{*}$. Indeed, for such $L_{*}$ there is $\mu \in C_{0}^{u}(\mathbb{G})^{*}$ positive such that, embedding $L^{1}(\mathbb{G})$ into $C_{0}^{u}(\mathbb{G})^{*}$, we have that $L_{*}$ is given by left multiplication by $\mu$. That is,

$$
\omega \circ L \circ \pi=(\mu \otimes \omega \circ \pi) \Delta=(\mu \otimes \omega)\left(\mathbb{W}^{*}(1 \otimes \pi(\cdot)) \mathbb{W}\right) .
$$

If $L_{*} \in \tilde{\mathbb{G}}$ then $L_{*}$ is a completely isometric isomorphism, so there exists a completely isometric $L_{*}^{-1}$. Thus $L_{*}^{-1}$ is also completely positive, and it is easy to see that $L_{*}^{-1}$ is also a left multiplier, compare the proof of [14, Theorem 4.7]. So choose $\mu^{-1} \in C_{0}^{u}(\mathbb{G})^{*}$ for $L_{*}^{-1}$. Both $L$ and $L^{-1}$ must be unital, completely positive, and so $\mu, \mu^{-1}$ are states.

For $x \in L^{\infty}(\mathbb{G}), \omega \in L^{1}(\mathbb{G})$,

$$
\begin{aligned}
\langle x, \omega\rangle & =\left\langle L^{-1}(L(x)), \omega\right\rangle=\left\langle\mu^{-1} \otimes \omega, \mathbb{W}^{*}(1 \otimes L(x)) \mathbb{W}\right\rangle \\
& =\left\langle\mu \otimes \mu^{-1} \otimes \omega, \mathbb{W}_{23}^{*} \mathbb{W}_{13}^{*}(1 \otimes 1 \otimes x) \mathbb{W}_{13} \mathbb{W}_{23}\right\rangle \\
& =\left\langle\left(\mu^{-1} \otimes \mu\right) \Delta \otimes \omega, \mathbb{W}^{*}(1 \otimes x) \mathbb{W}\right\rangle,
\end{aligned}
$$

as $\mathbb{W}$ is a corepresentation of $C_{0}^{u}(\mathbb{G})$. Apply this to $x=(\mathrm{id} \otimes \widehat{\omega})(W)$ to see that

$$
x=\left(\mu^{-1} \star \mu \otimes \mathrm{id}\right)\left(\mathbb{W}^{*}(1 \otimes x) \mathbb{W}\right)=\left(\mu^{-1} \star \mu \otimes \mathrm{id} \otimes \widehat{\omega}\right)\left(\mathbb{W}_{12}^{*} W_{23} \mathbb{W}_{12}\right) .
$$

As $\mathbb{W}_{12}^{*} W_{23} \mathbb{W}_{12}=((\mathrm{id} \otimes \pi) \Delta \otimes \mathrm{id})(\mathbb{W})=\mathbb{W}_{13} W_{23}$ it follows that

$$
(\mathrm{id} \otimes \widehat{\omega})(W)=\left(\mu^{-1} \star \mu \otimes \mathrm{id} \otimes \widehat{\omega}\right)\left(\mathbb{W}_{13} W_{23}\right),
$$

and so $W=\left(\mu^{-1} \star \mu \otimes \mathrm{id} \otimes \mathrm{id}\right)\left(\mathbb{W}_{13} W_{23}\right)$ and so $\left(\mu^{-1} \star \mu \otimes \mathrm{id}\right)(\mathbb{W})=1$. Similarly $\left(\mu \star \mu^{-1} \otimes \mathrm{id}\right)(\mathbb{W})=$ 1. By [17, Proposition 6.3] and its proof, it follows that $\mu^{-1} \star \mu=\mu \star \mu^{-1}=\epsilon$ the counit of $C_{0}^{u}(\mathbb{G})$.

Let $T=(\mu \otimes \mathrm{id}) \Delta: C_{0}^{u}(\mathbb{G}) \rightarrow C_{0}^{u}(\mathbb{G})$, a unital completely positive map. That $T$ maps into $C_{0}^{u}(\mathbb{G})$, and not $M\left(C_{0}^{u}(\mathbb{G})\right)$, follows by observing that $\left\{(\mathrm{id} \otimes \omega)(\mathbb{W}): \omega \in L^{1}(\widehat{\mathbb{G}})\right\}$ is norm dense in $C_{0}^{u}(\mathbb{G})$, see the discussion after $[17$, Proposition 5.1], and then calculating that

$$
T((\mathrm{id} \otimes \omega)(\mathbb{W}))=(\mu \otimes \mathrm{id} \otimes \omega)\left(\mathbb{W}_{13} \mathbb{W}_{23}\right)=(\mathrm{id} \otimes \omega a)(\mathbb{W}) \in C_{0}^{u}(\mathbb{G}),
$$

where $a=(\mu \otimes \mathrm{id})(\mathrm{W}) \in L^{\infty}(\widehat{\mathbb{G}})$. We similarly form $T^{-1}$, and observe that $T^{-1}$ is the inverse of $T$. Indeed,

$$
\begin{aligned}
T^{-1}(T(x)) & =\left(\mu^{-1} \otimes \mathrm{id}\right) \Delta((\mu \otimes \mathrm{id}) \Delta(x))=\left(\mu^{-1} \otimes \mu \otimes \mathrm{id}\right) \Delta^{2}(x) \\
& =\left(\left(\mu^{-1} \otimes \mu\right) \Delta \otimes \mathrm{id}\right) \Delta(x)=(\epsilon \otimes \mathrm{id}) \Delta(x)=x .
\end{aligned}
$$

We now use the Schwarz inequality, and the theory of multiplicative domains, for completely positive maps, see [4, Proposition 1.5.7] for example. For $a \in C_{0}^{u}(\mathbb{G})$,

$$
a^{*} a=T^{-1}(T(a))^{*} T^{-1}(T(a)) \leq T^{-1}\left(T(a)^{*} T(a)\right) \leq T^{-1}\left(T\left(a^{*} a\right)\right)=a^{*} a,
$$

and so we have equality throughout, namely $a^{*} a=T^{-1}\left(T(a)^{*} T(a)\right)$ or equivalently, $T\left(a^{*} a\right)=$ $T(a)^{*} T(a)$. Similarly we can show that $T\left(a a^{*}\right)=T(a) T(a)^{*}$, and it hence follows that $T(a b)=$ $T(a) T(b)$ for all $a, b \in C_{0}^{u}(\mathbb{G})$. Thus $T$ is a $*$-automorphism of $C_{0}^{u}(\mathbb{G})$. As $\mu=\epsilon \circ T$ it follows that $\mu$ is a character.

Then let $\widehat{u}=(\mu \otimes \mathrm{id})(\mathbb{W})$ so $\widehat{\Delta}(\widehat{u})=(\mu \otimes \mathrm{id} \otimes \mathrm{id})\left(\mathbb{W}_{13} \mathbb{W}_{12}\right)=\widehat{u} \otimes \widehat{u}$ as $\mu$ is multiplicative, so $\widehat{u} \in \operatorname{Gr}(\widehat{\mathbb{G}})$. Then, for $x \in L^{\infty}(\mathbb{G})$,

$$
L(x)=(\mu \otimes \mathrm{id})\left(\mathrm{W}^{*}(1 \otimes x) \mathrm{W}\right)=\widehat{u}^{*} x \widehat{u},
$$


as claimed. Finally, that $\widehat{\Delta}(\widehat{u})=\widehat{u} \otimes \widehat{u}$ is equivalent to $\widehat{W}^{*}(1 \otimes \widehat{u}) \widehat{W}=\widehat{u} \otimes \widehat{u}$ or equivalently that $W(\widehat{u} \otimes 1) W^{*}=\widehat{u} \otimes \widehat{u}$, and so

$$
(L \otimes \mathrm{id})(W)=\left(\widehat{u}^{*} \otimes 1\right) W(\widehat{u} \otimes 1)=\left(\widehat{u}^{*} \otimes 1\right)(\widehat{u} \otimes \widehat{u}) W=(1 \otimes \widehat{u}) W,
$$

and so $\widehat{u} \in M_{c b}^{l}\left(L^{1}(\mathbb{G})\right)$ is associated to $L$ as required.

Notice that the previous proof did not use weight theory (and that neither does [7]). We now show that our maps are homeomorphisms: this is a new result for the equivalence with $\operatorname{Gr}_{u}(\widehat{\mathbb{G}})$, and for completeness, we give a complete proof. As $\tilde{\mathbb{G}}$ is easily seen to be a topological group, [16, Proposition 3.5], and $\operatorname{sp}\left(C_{0}^{u}(\mathbb{G})\right)$ is locally compact, it follows that the intrinsic group is indeed a locally compact group. Our proof will avoid use of weight theory, standard position of von Neumann algebras etc., compare the proof of [16, Theorem 3.7].

Theorem 4.4. The bijections between our four equivalent conditions are homeomorphisms.

Proof. Firstly, the map $\operatorname{sp}\left(C_{0}^{u}(\mathbb{G})\right) \rightarrow \operatorname{Gr}(\widehat{\mathbb{G}}) ; \gamma \mapsto(\gamma \otimes \mathrm{id})(\mathbb{W})$ is a homeomorphism. This follows, as $\gamma_{i} \rightarrow \gamma$ is equivalent to $\lim _{i} \gamma_{i}(a)=\gamma(a)$ for all $a \in C_{0}^{u}(\mathbb{G})$ of the form $(\mathrm{id} \otimes \widehat{\omega})(\mathbb{W})$ for $\widehat{\omega} \in L^{1}(\widehat{\mathbb{G}})$, as such $a$ are norm dense, and $\left(\gamma_{i}\right)$ is a bounded net. However, this is clearly equivalent to $\left(\gamma_{i} \otimes \mathrm{id}\right)(\mathbb{W}) \rightarrow(\gamma \otimes \mathrm{id})(\mathbb{W})$ weak $^{*}$ in $L^{\infty}(\widehat{\mathbb{G}})$, as required.

We next show that $\widetilde{\mathbb{G}} \rightarrow \operatorname{Gr}_{u}(\widehat{\mathbb{G}})$ is continuous. Let the (bounded) net $\left(L_{i, *}\right) \subseteq \tilde{\mathbb{G}} \subseteq C_{c b}^{l}\left(L^{1}(\mathbb{G})\right)$ converge strongly to $L_{*}$, and be associated to $\widehat{u}_{i} \in \operatorname{Gr}_{u}(\widehat{\mathbb{G}})$, with $L_{*}$ associated to $\widehat{u}$. As $\left(\widehat{u}_{i}\right)$ is a net of unitaries, to show that $\widehat{u}_{i} a \rightarrow \widehat{u} a$ for each $a \in C_{0}^{u}(\widehat{\mathbb{G}})$, it suffices to check for a dense collection of such $a$, for example, $a=(\omega \otimes \mathrm{id})(\mathrm{W})$ for $\omega \in L^{1}(\mathbb{G})$. However,

$$
\begin{aligned}
\lim _{i} \widehat{u}_{i}(\omega \otimes \mathrm{id})(\mathrm{W}) & =\lim _{i}(\omega \otimes \mathrm{id})\left(\left(1 \otimes \widehat{u}_{i}\right) \mathrm{W}\right)=\lim _{i}(\omega \otimes \mathrm{id})\left(L_{i} \otimes \mathrm{id}\right)(\mathrm{W}) \\
& =\lim _{i}\left(L_{i, *}(\omega) \otimes \mathrm{id}\right)(\mathrm{W})=\left(L_{*}(\omega) \otimes \mathrm{id}\right)(\mathrm{W})=\widehat{u}(\omega \otimes \mathrm{id})(\mathrm{W}),
\end{aligned}
$$

as required. We similarly need to show that $\widehat{u}_{i}^{*}(\omega \otimes \mathrm{id})(\mathrm{W}) \rightarrow \widehat{u}^{*}(\omega \otimes \mathrm{id})(\mathrm{W})$ for each $\omega$. However, as $\widehat{u}_{i}^{*}=\widehat{u}_{i}^{-1}$, this claim will follow because $L_{i, *}^{-1} \rightarrow L_{*}^{-1}$. Thus $\widehat{u}_{i} \rightarrow \widehat{u}$ strictly, as claimed.

That $\operatorname{Gr}_{u}(\widehat{\mathbb{G}}) \rightarrow \operatorname{Gr}(\widehat{\mathbb{G}})$ is continuous follows easily, as $\pi$ is strictly continuous, and strict convergence in $M\left(C_{0}(\widehat{\mathbb{G}})\right)$ implies weak*-convergence in $L^{\infty}(\widehat{\mathbb{G}})$.

Finally we show that $\operatorname{Gr}(\widehat{\mathbb{G}}) \rightarrow \tilde{\mathbb{G}}$ is continuous. Continuing with the same notation, suppose that $\widehat{u}_{i} \rightarrow \widehat{u}$ weak $^{*}$ in $\operatorname{Gr}(\widehat{\mathbb{G}})$. As each $\widehat{u}_{i}$ is unitary, this implies that actually $\widehat{u}_{i} \rightarrow \widehat{u}$ strongly, in $\mathcal{B}\left(L^{2}(\mathbb{G})\right)$. This then implies that for all $\xi, \eta \in L^{2}(\mathbb{G})$,

$$
\lim _{i} \widehat{u}_{i} \omega_{\xi, \eta} \widehat{u}_{i}^{*}=\lim _{i} \omega_{\widehat{u}_{i} \xi, \widehat{u}_{i} \eta}=\omega_{\widehat{u} \xi, \widehat{u} \eta}=\widehat{u} \omega_{\xi, \eta} \widehat{u}^{*}
$$

in $\mathcal{B}\left(L^{2}(\mathbb{G})\right)_{*}$ and hence also in $L^{1}(\mathbb{G})$. However, as $L_{i}(x)=\widehat{u}_{i}^{*} x \widehat{u}_{i}$, this shows that $L_{i, *}\left(\omega_{\xi, \eta}\right) \rightarrow$ $L_{*}\left(\omega_{\xi, \eta}\right)$ in $L^{1}(\mathbb{G})$. As $\left(L_{i, *}\right)$ is a bounded net, it follows that $L_{i, *} \rightarrow L_{*}$ strongly, as required.

\section{The Intrinsic Group functor}

In this section, we shall show that the assignment $\mathbb{G} \rightarrow \tilde{\mathbb{G}}$ is actually a functor. Given the results of Section 3.2, we have little choice as to how a morphism $\mathbb{G} \rightarrow \mathbb{H}$ should map $\tilde{\mathbb{G}}$ to $\tilde{\mathbb{H}}$, as $\tilde{\mathbb{G}}$ is realised as a subset of the multipliers of $L^{1}(\mathbb{G})$. Fortunately, this works!

Theorem 5.1. Let $f: \mathbb{G} \rightarrow \mathbb{H}$ be a morphism of quantum groups, which induces the completely contractive homomorphism $C_{c b}^{l}\left(L^{1}(\mathbb{G})\right) \rightarrow C_{c b}^{l}\left(L^{1}(\mathbb{H})\right)$, as before. This restricts to a continuous group homomorphism $\tilde{f}: \tilde{\mathbb{G}} \rightarrow \tilde{\mathbb{H}}$. The assignment $f \mapsto \tilde{f}$ is a functor. 
Indeed, let the morphism be represented by $\phi: C_{0}^{u}(\mathbb{H}) \rightarrow M\left(C_{0}^{u}(\mathbb{G})\right)$ and $\beta: C_{0}(\mathbb{H}) \rightarrow$ $M\left(C_{0}(\mathbb{G}) \otimes C_{0}(\mathbb{H})\right)$, with dual counterparts $\widehat{\phi}$ and $\widehat{\beta}$. Let $L_{*} \in \tilde{\mathbb{G}}$ be associated to $\gamma \in \operatorname{sp}\left(C_{0}^{u}(\mathbb{G})\right), \widehat{u} \in$ $\operatorname{Gr}(\widehat{\mathbb{G}})$ and $\widehat{u}_{u} \in \operatorname{Gr}_{u}(\widehat{\mathbb{G}})$, and let $L_{*}$ be mapped to $L_{*}^{\prime} \in \tilde{\mathbb{H}}$, associated to $\gamma^{\prime}, \widehat{u}^{\prime}, \widehat{u}_{u}^{\prime}$. Then we have the following relations:

- $\widehat{u}_{u}^{\prime}=\widehat{\phi}\left(\widehat{u}_{u}\right)$;

- $\widehat{\beta}(\widehat{u})=\widehat{u}^{\prime} \otimes \widehat{u}$;

- $\beta L^{\prime}=(L \otimes \mathrm{id}) \beta$;

- $\gamma^{\prime}=\gamma \circ \phi$.

Proof. By definition, the map $C_{c b}^{l}\left(L^{1}(\mathbb{G})\right) \rightarrow C_{c b}^{l}\left(L^{1}(\mathbb{H})\right)$ is induced by the restriction of $\widehat{\phi}$ to a $\operatorname{map} M_{c b}^{l, u}\left(L^{1}(\mathbb{G})\right) \rightarrow M_{c b}^{l, u}\left(L^{1}(\mathbb{H})\right)$, see Theorem 3.6. As $\widehat{\phi}$ is a Hopf $*$-homomorphism, it's clear that $\widehat{\phi}\left(\widehat{u}_{u}\right) \in \operatorname{Gr}_{u}(\widehat{\mathbb{H}})$ for each $\widehat{u}_{u} \in \operatorname{Gr}_{u}(\widehat{\mathbb{G}})$. So we do obtain a map $\tilde{f}: \tilde{\mathbb{G}} \rightarrow \tilde{\mathbb{H}}$. As the product on $\operatorname{Gr}_{u}(\widehat{\mathbb{G}})$ is simply the restriction of the product on $M\left(C_{0}^{u}(\mathbb{G})\right)$, and as $\widehat{\phi}$ is a homomorphism, the map $\tilde{f}: \tilde{\mathbb{G}} \rightarrow \tilde{\mathbb{H}}$ is a group homomorphism, clearly continuous. Finally, because composition of morphisms is given by composition of the associated Hopf $*$-homomorphisms, it is clear that $f \mapsto \tilde{f}$ is a functor, namely that if $h=g \circ f$ then $\tilde{h}=\tilde{g} \circ \tilde{f}$.

By Lemma 3.9 and Theorem 4.3 , we then see that

$$
\left(1 \otimes \widehat{u}^{\prime}\right) U=(L \otimes \mathrm{id})(U)=\left(\widehat{u}^{*} \otimes 1\right) U(\widehat{u} \otimes 1)
$$

and so, as $\widehat{U}=\sigma\left(U^{*}\right)$, it follows that

$$
\left(\widehat{u}^{\prime} \otimes 1\right) \widehat{U}^{*}=\left(1 \otimes \widehat{u}^{*}\right) \widehat{U}^{*}(1 \otimes \widehat{u}),
$$

which in turn implies that

$$
\widehat{\beta}(\widehat{u})=\widehat{U}^{*}(1 \otimes \widehat{u}) \widehat{U}=\widehat{u}^{\prime} \otimes \widehat{u},
$$

as claimed.

That $\beta L^{\prime}=(L \otimes \mathrm{id}) \beta$ follows immediately from Corollary 3.11 , and that $\gamma^{\prime}=\gamma \circ \phi$ follows immediately from Proposition 3.8 .

\subsection{Universal property}

In this section, we shall construct a morphism $\tilde{\mathbb{G}} \rightarrow \mathbb{G}$, and show that $\tilde{\mathbb{G}}$ satisfies a natural universal property. We then draw some category theoretic conclusions. In the next section, we shall show that actually $\tilde{\mathbb{G}}$ is a "closed quantum subgroup" of $\mathbb{G}$.

Let us view $\tilde{\mathbb{G}}$ as being $\operatorname{Gr}_{u}(\widehat{\mathbb{G}})$ with the strict topology. Thus the formal identity map $\tilde{\mathbb{G}} \rightarrow$ $M\left(C_{0}^{u}(\widehat{\mathbb{G}})\right)$ is a continuous homomorphism, when $M\left(C_{0}^{u}(\widehat{\mathbb{G}})\right)$ carries the strict topology. If $C_{0}^{u}(\widehat{\mathbb{G}}) \subseteq$ $\mathcal{B}(H)$ is a faithful non-degenerate *-representation, then $M\left(C_{0}^{u}(\mathbb{G})\right) \subseteq \mathcal{B}(H)$ as well, and the induced map $\tilde{\mathbb{G}} \rightarrow \mathcal{B}(H)$ will be a strongly continuous unitary representation. By the universal property of $C^{*}(\tilde{\mathbb{G}})$, we hence obtain a $*$-homomorphism $C^{*}(\tilde{\mathbb{G}}) \rightarrow \mathcal{B}(H)$; and one can show that this takes values in $M\left(C_{0}^{u}(\widehat{\mathbb{G}})\right)$. So we obtain a non-degenerate $*$-homomorphism $\widehat{\theta}: C^{*}(\tilde{\mathbb{G}}) \rightarrow$ $M\left(C_{0}^{u}(\widehat{\mathbb{G}})\right)$. The strict extension of this map sends an element of $\tilde{\mathbb{G}}$ to its image in $\operatorname{Gr}_{u}(\widehat{\mathbb{G}})$, and thus by the definition of the coproduct on $C^{*}(\tilde{\mathbb{G}})$, we see that $\widehat{\theta}$ is a Hopf $*$-homomorphism. Hence we have a morphism $\widehat{\mathbb{G}} \rightarrow \widehat{\widetilde{G}}$ and so by duality, the claimed morphism $\widetilde{\mathbb{G}} \rightarrow \mathbb{G}$.

We constructed this morphism from what might be called a group representation perspective. The following shows that it also has an extremely natural interpretation at the $C^{*}$-algebra level. 
Proposition 5.2. Let the morphism $\tilde{\mathbb{G}} \rightarrow \mathbb{G}$ induce the Hopf $*$-homomorphism $\theta: C_{0}^{u}(\mathbb{G}) \rightarrow$ $M\left(C_{0}(\tilde{\mathbb{G}})\right)$. Then $\theta$ maps into $C_{0}(\tilde{\mathbb{G}})$, and is surjective. Viewing $\tilde{\mathbb{G}}$ as $\operatorname{sp}\left(C_{0}^{u}(\mathbb{G})\right)$, the map $\theta$ is nothing but the Gelfand transform.

Proof. For a good treatment of Gelfand transforms for non-unital algebras, see [6, Section 2.3], in particular [6, Theorem 2.3.25]. In keeping with our notation, let $C_{0}^{u}(\mathbb{G}) \rightarrow C_{0}(\tilde{\mathbb{G}}) ; a \mapsto \tilde{a}$ be the Gelfand transform, so that $\tilde{a}(\gamma)=\langle\gamma, a\rangle$ for $a \in C_{0}^{u}(\mathbb{G}), \gamma \in \tilde{\mathbb{G}}=\operatorname{sp}\left(C_{0}^{u}(\mathbb{G})\right)$. Then the algebra $\left\{\tilde{a}: a \in C_{0}^{u}(\mathbb{G})\right\}$ is self-adjoint, separates the points of $\tilde{\mathbb{G}}$, and separates the points from 0 , and hence is dense in $C_{0}(\tilde{\mathbb{G}})$. We conclude that the Gelfand transform is onto.

It hence remains to show that $\theta(a)=\tilde{a}$ for each $a \in C_{0}^{u}(\mathbb{G})$. Consider the universal bicharacter for $\tilde{\mathbb{G}}$. This is

$$
\mathbb{W}_{\tilde{\mathbb{G}}}=\mathrm{W}_{\tilde{\mathbb{G}}} \in M\left(C_{0}(\tilde{\mathbb{G}}) \otimes C^{*}(\tilde{\mathbb{G}})\right)=C_{b}^{s t r}\left(\tilde{\mathbb{G}}, M\left(C^{*}(\tilde{\mathbb{G}})\right)\right),
$$

the space of bounded strictly continuous maps $\tilde{\mathbb{G}} \rightarrow M\left(C^{*}(\tilde{\mathbb{G}})\right)$, and under this identification, $\mathbb{W}_{\tilde{\mathbb{G}}}$ is nothing but the inclusion $\tilde{\mathbb{G}} \rightarrow M\left(C^{*}(\tilde{\mathbb{G}})\right)$. By definition,

$$
(\theta \otimes \mathrm{id})\left(\mathbb{W}_{\mathbb{G}}\right)=(\operatorname{id} \otimes \widehat{\theta})\left(\mathbb{W}_{\tilde{\mathbb{G}}}\right) \in C_{b}^{s t r}\left(\tilde{\mathbb{G}}, M\left(C_{0}^{u}(\widehat{\mathbb{G}})\right)\right),
$$

and by the construction of $\widehat{\theta}$, this is the inclusion $\widetilde{\mathbb{G}}=\operatorname{Gr}_{u}(\widehat{\mathbb{G}}) \rightarrow M\left(C_{0}^{u}(\widehat{\mathbb{G}})\right)$. Let $\gamma \in \operatorname{sp}\left(C_{0}^{u}(\mathbb{G})\right)$ be associated to $\widehat{u}_{u} \in \mathrm{Gr}_{u}(\widehat{\mathbb{G}})$. Viewing $(\theta \otimes \mathrm{id})\left(\mathbb{W}_{\mathbb{G}}\right)$ as a strictly continuous function $\widetilde{\mathbb{G}} \rightarrow M\left(C_{0}^{u}(\widehat{\mathbb{G}})\right)$, the value of this function at $\gamma$ is hence simply $\widehat{u}_{u}$. However, this is equal to $(\gamma \otimes \mathrm{id})\left(\mathbb{W}_{\mathbb{G}}\right)$.

So, we have that $(\theta \otimes \mathrm{id})\left(\mathbb{W}_{\mathbb{G}}\right) \in M\left(C_{0}(\widetilde{\mathbb{G}}) \otimes C_{0}^{u}(\widehat{\mathbb{G}})\right)=C_{b}^{s t r}\left(\tilde{\mathbb{G}}, M\left(C_{0}^{u}(\widehat{\mathbb{G}})\right)\right)$ is the function $\gamma \mapsto(\gamma \otimes \mathrm{id})\left(\mathbb{W}_{\mathbb{G}}\right)$. Apply id $\otimes \pi_{\widehat{\mathbb{G}}}$ and then slice by some $\widehat{\omega} \in L^{1}(\widehat{\mathbb{G}})$ to see that $\theta((\mathrm{id} \otimes \widehat{\omega})(\mathbb{W})) \in$ $M\left(C_{0}(\tilde{\mathbb{G}})\right)$ is the function

$$
\gamma \mapsto\langle\gamma,(\operatorname{id} \otimes \widehat{\omega})(\mathbb{W})\rangle .
$$

As the collection of elements $(\mathrm{id} \otimes \widehat{\omega})(\mathrm{W})$ is dense in $C_{0}^{u}(\mathbb{G})$, we conclude that $\theta$ is indeed nothing but the Gelfand transform.

That $\theta$ is a surjection $C_{0}^{u}(\widehat{\mathbb{G}}) \rightarrow C_{0}(\tilde{\mathbb{G}})$ means that $\tilde{\mathbb{G}}$ is identified as a Woronowicz closed quantum subgroup of $\mathbb{G}$, see [10]. In the next section, we shall prove that $\tilde{\mathbb{G}}$ satisfies the a priori stronger condition of being Vaes closed.

We also note that if the reader is unhappy with the slightly sketchy proof just given, then we could simply define $\theta$ to be the Gelfand transform: it is very easy to show that $\theta$ is then a Hopf *-homomorphism. However, we feel that for motivational purposes, the definition of $\widehat{\theta}$ is more natural.

The following shows that $\tilde{\mathbb{G}}$ is then the maximal "classical" subgroup of $\mathbb{G}$.

Theorem 5.3. The morphism $\tilde{\mathbb{G}} \rightarrow \mathbb{G}$ satisfies the following universal property. For any locally compact group $H$, and any morphism $H \rightarrow \mathbb{G}$, there is a unique continuous group homomorphism $H \rightarrow \tilde{\mathbb{G}}$ making the following, equivalent, diagrams commute:
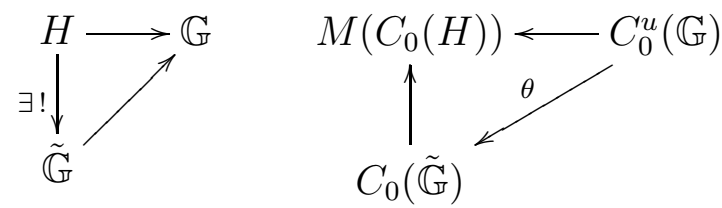

Proof. That the two diagrams are equivalent follows from the definition of what a morphism of locally compact quantum groups is. As $\theta$ is onto, if a Hopf $*$-homomorphism $C_{0}(\tilde{\mathbb{G}}) \rightarrow M\left(C_{0}(H)\right)$ exists, making the diagram commute, then it is uniquely defined. 
Let $\phi: C_{0}^{u}(\mathbb{G}) \rightarrow M\left(C_{0}(H)\right)$ be our Hopf $*$-homomorphism. For each $h \in H$ let $\delta_{h}: C_{0}(H) \rightarrow \mathbb{C}$ be the functional given by point evaluation. So $\delta_{h}$ is a character, and as $\phi$ is non-degenerate, $\delta_{h} \circ \phi$ is a character, and hence defines a member of $\widetilde{\mathbb{G}}$. We hence obtain our map $H \rightarrow \tilde{\mathbb{G}}$, which is easily seen to be continuous, and thus induces $\psi: C_{0}(\tilde{\mathbb{G}}) \rightarrow M\left(C_{0}(H)\right)$. Then, for $a \in C_{0}^{u}(\mathbb{G})$ and $h \in H$,

$$
\psi(\theta(a))(h)=\theta(a)\left(\delta_{h} \circ \phi\right)=\left\langle\delta_{h} \circ \phi, a\right\rangle=\phi(a)(h),
$$

and so $\psi \circ \theta=\phi$ as required. As $\theta, \phi$ are Hopf $*$-homomorphisms,

$$
\Delta_{H} \psi \theta=\Delta_{H} \phi=(\phi \otimes \phi) \Delta_{\mathbb{G}}=(\psi \theta \otimes \psi \theta) \Delta_{\mathbb{G}}=(\psi \otimes \psi) \Delta_{\widetilde{G}} \theta .
$$

As $\theta$ is onto, it follows that $\psi$ is a Hopf $*$-homomorphism, and so our map $H \rightarrow \tilde{\mathbb{G}}$ is a group homomorphism, as required.

We can of course dualise this universal property, and obtain two more commuting diagrams

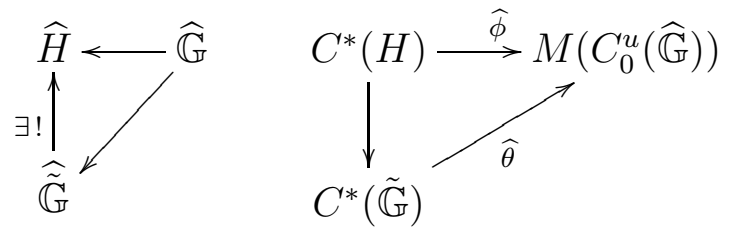

As $\widehat{\phi}: C^{*}(H) \rightarrow M\left(C_{0}^{u}(\widehat{\mathbb{G}})\right)$ is a Hopf $*$-homomorphism, the strict extension must send $h \in H \subseteq$ $M\left(C^{*}(H)\right)$ to a member of $\operatorname{Gr}_{u}(\widehat{\mathbb{G}})=\tilde{\mathbb{G}}$, and this gives the map $C^{*}(H) \rightarrow C^{*}(\tilde{\mathbb{G}})$.

Let us think about this result from the perspective of some elementary category theory. Let LCG and LCQG be the categories of locally compact groups and locally compact quantum groups, respectively. The definition of a morphism in LCQG is setup precisely so that the assignment of $G \in \mathrm{LCG}$ to $\left(C_{0}(G), \Delta_{G}\right) \in \mathrm{LCQG}$ is a functor. Let this functor be $\mathcal{C}: \mathrm{LCG} \rightarrow \mathrm{LCQG}$, we choose $\mathcal{C}$ for "classical". Let $\mathcal{I}:$ LCQG $\rightarrow$ LCG be the "intrinsic group functor", the assignment of $\tilde{\mathbb{G}}$ to $\mathbb{G}$. We recall the notion of an adjoint functor, see for example [22, Chapter 2].

Theorem 5.4. The functor $\mathcal{I}$ is a right adjoint to the functor $\mathcal{C}$.

Proof. This is equivalent to $\mathcal{C}$ being a left adjoint to $\mathcal{I}$. There are a number of different, equivalent meanings to this. One is that $\mathcal{C}: L C Q G \leftarrow L C G$ is a left adjoint functor if for each $\mathbb{G} \in L C Q G$ there is a terminal morphism from $\mathcal{C}$ to $\mathbb{G}$. That is, there exists $\tilde{\mathbb{G}} \in \mathrm{LCG}$ and $\mathcal{C}(\tilde{\mathbb{G}}) \rightarrow \mathbb{G}$ such that for each $H \in \mathrm{LCG}$ and each morphism $f: \mathcal{C}(H) \rightarrow \mathbb{G}$, there is a unique morphism $H \rightarrow \widetilde{\mathbb{G}}$ with

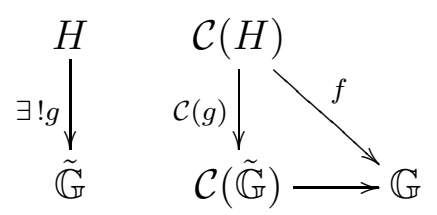

However, if we remember that $\mathcal{C}$ is essentially the "inclusion", then this is nothing but the universal property of $\tilde{\mathbb{G}}$ just shown in Theorem 5.3. In this situation, it is then actually automatic (from purely category theoretic considerations) that $\mathcal{I}: \mathbb{G} \rightarrow \tilde{\mathbb{G}}$ is a functor (the universal property alone can be used to construct $\mathcal{I}(f): \tilde{\mathbb{G}} \rightarrow \tilde{\mathbb{H}}$ given any $f: \mathbb{G} \rightarrow \mathbb{H})$.

\subsection{Closed subgroups}

In [10] the notion of a closed quantum subgroup was explored. We say that a morphism $\mathbb{H} \rightarrow \mathbb{G}$ identifies $\mathbb{H}$ as a closed quantum subgroup of $\mathbb{G}$, in the sense of Woronowicz, if the Hopf *homomorphism $C_{0}^{u}(\mathbb{G}) \rightarrow M\left(C_{0}^{u}(\mathbb{H})\right)$ maps into, and onto, $C_{0}^{u}(\mathbb{H})$. This notion is easily seen to 
reduce to the classical notion of a closed group, when applied to a classical group $G$, see [10, Sections 3,4].

When $H \subseteq G$ is a closed subgroup of a locally compact group, the Herz Restriction Theorem (see [12]) tells us that the restriction map gives a quotient map from the Fourier algebra $A(G)$ to $A(H)$, or equivalently, gives a normal injective $*$-homomorphism $V N(H) \rightarrow V N(G)$. This notion was generalised to quantum groups in [28, Definition 2.5], and motivated the authors of [10] to give the notion of a Vaes closed quantum subgroup. Here we state the original definition: $\mathbb{H} \rightarrow \mathbb{G}$ identifies $\mathbb{H}$ as a Vaes closed quantum subgroup of $\mathbb{G}$ if there is a normal, unital, injective *-homomorphism $\gamma: L^{\infty}(\widehat{\mathbb{H}}) \rightarrow L^{\infty}(\widehat{\mathbb{G}})$ with $\pi_{\widehat{\mathbb{G}}} \circ \widehat{\phi}=\gamma \circ \pi_{\widehat{\mathbb{H}}}$ where $\widehat{\phi}: C_{0}^{u}(\widehat{\mathbb{H}}) \rightarrow M\left(C_{0}^{u}(\widehat{\mathbb{G}})\right)$ is the Hopf $*$-homomorphism representing the dual morphism $\widehat{\mathbb{G}} \rightarrow \widehat{\mathbb{H}}$.

We observed above that $\tilde{\mathbb{G}}$ is Woronowicz closed in $\mathbb{G}$. In [16, Theorem 3.14] it's shown that $\mathbb{G} \rightarrow$ $\tilde{\mathbb{G}}$ preserves compactness and discreteness. As $\tilde{\mathbb{G}}$ is a closed quantum subgroup of $\mathbb{G}$, this result now also follows immediately from results of $[10]$. Indeed, if $\mathbb{G}$ is compact, then $C_{0}^{u}(\mathbb{G})$ is unital, so $C_{0}(\tilde{\mathbb{G}})$ is unital, so $\tilde{\mathbb{G}}$ is compact. The discrete case is more involved, see [10, Theorem 6.2].

We now prove a stronger result, that $\tilde{\mathbb{G}}$ is Vaes closed.

Theorem 5.5. The morphism $\theta: C_{0}^{u}(\widehat{\mathbb{G}}) \rightarrow C_{0}(\tilde{\mathbb{G}})$ identifies $\tilde{\mathbb{G}}$ as a Vaes closed quantum subgroup of $\mathbb{G}$.

Proof. Consider the dual morphism $\widehat{\theta}: C^{*}(\tilde{\mathbb{G}}) \rightarrow M\left(C_{0}^{u}(\widehat{\mathbb{G}})\right)$. Compose with $\pi_{\widehat{\mathbb{G}}}$ and let $M$ be the von Neumann algebra generated by the image in $L^{\infty}(\widehat{\mathbb{G}})$. As $\pi_{\widehat{\mathbb{G}}} \circ \widehat{\theta}$ is a Hopf $*$-homomorphism, it follows by weak*-continuity that $\Delta_{\widehat{\mathbb{G}}}(x) \in M \bar{\otimes} M \subseteq L^{\infty}(\widehat{\mathbb{G}}) \bar{\otimes} L^{\infty}(\widehat{\mathbb{G}})$ for each $x \in M$. By [17, Remark 12.1] we also know that $\widehat{\theta}$ intertwines the scaling group $\left(\tau_{t}\right)$ and the unitary antipode $R$; the same is true of $\pi_{\widehat{G}}$. It follows that $\left(\tau_{t}\right)$ restricts to the identity map on $M$, and that $R$ restricts to $M$. It follows from [2, Proposition A5], that $M$ "is a quantum group", that is, admits invariant weights. For us, an convenient way to restate this is that there is a locally compact quantum group $\mathbb{K}$ and a normal $*$-isomorphism $\psi: L^{\infty}(\mathbb{K}) \rightarrow M \subseteq L^{\infty}(\widehat{\mathbb{G}})$ with $\psi$ intertwining the coproducts of $\mathbb{K}$ and $\widehat{\mathbb{G}}$. As $C^{*}(\tilde{\mathbb{G}})$ is cocommutative and its image is weak ${ }^{*}$-dense in $L^{\infty}(\mathbb{K})$, it follows that $\mathbb{K}$ is cocommutative, and so there is a locally compact group $K$ with $L^{\infty}(\mathbb{K})=V N(K)$.

Thus, we obtain the following commutative diagram:

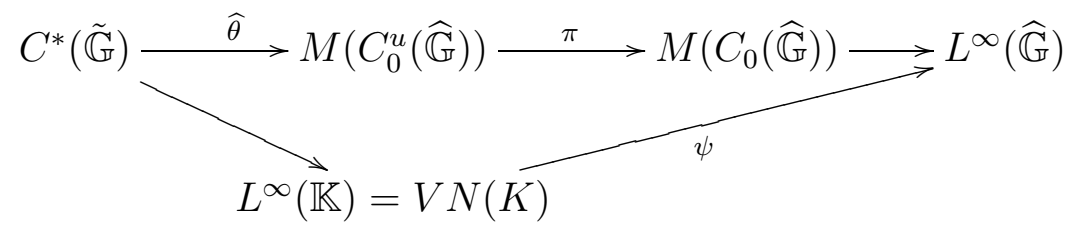

where $\psi$ is a normal $*$-isomorphism onto its range, which intertwines the coproducts. This means, in particular, that $\psi$ restricts to a map $K=\operatorname{Gr}(V N(K)) \rightarrow \operatorname{Gr}(\widehat{\mathbb{G}})=\widetilde{\mathbb{G}}$, and so we obtain an injective group homomorphism $f: K \rightarrow \tilde{\mathbb{G}}$. As $\psi$ is normal, by the definition of the topologies on $\operatorname{Gr}(V N(K))$ and $\operatorname{Gr}(\widehat{\mathbb{G}})$, we see that $f$ is continuous.

It follows from the discussion in Section 5.1 that, if we regard $\tilde{\mathbb{G}}$ as being the set $\operatorname{Gr}(\widehat{\mathbb{G}})$, then the map $\pi \widehat{\theta}$ sends $\widehat{u} \in \tilde{\mathbb{G}} \subseteq M\left(C^{*}(\tilde{\mathbb{G}})\right)$ to $\widehat{u} \in \operatorname{Gr}(\widehat{\mathbb{G}}) \subseteq L^{\infty}(\widehat{\mathbb{G}})$. As such $\widehat{u} \in M$, we see that $\psi^{-1}(\widehat{u}) \in \operatorname{Gr}(V N(K))$, and so we obtain again a continuous group homomorphism $g: \tilde{\mathbb{G}} \rightarrow K$. Then $f \circ g: \tilde{\mathbb{G}} \rightarrow \tilde{\mathbb{G}}$ is the identity, and as $f$ is injective, $f$ and $g$ are mutual inverses.

Thus $K \cong \tilde{\mathbb{G}}$ and so $V N(K) \cong V N(\tilde{\mathbb{G}})$. The isomorphisms involved ensure that the induced map $\theta_{0}: V N(\tilde{\mathbb{G}}) \rightarrow L^{\infty}(\widehat{\mathbb{G}})$ restricts to the identity map on $\tilde{\mathbb{G}}=\operatorname{Gr}(\widehat{\mathbb{G}})$. Hence we obtain the 
following commutative diagram:

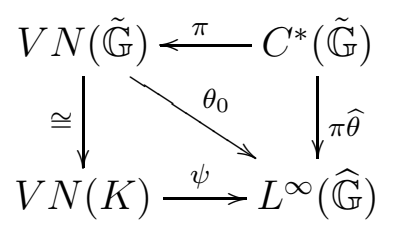

as required.

We have hence obtained a normal injective unital $*$-homomorphism $\theta_{0}: V N(\widetilde{\mathbb{G}}) \rightarrow L^{\infty}(\widehat{\mathbb{G}})$ such $\pi_{\widehat{\mathbb{G}}} \widehat{\theta}=\theta_{0} \pi_{\widehat{\widetilde{G}}}$. By the definition of $\widehat{\theta}$, it follows that if we identify $\tilde{\mathbb{G}}$ with $\operatorname{Gr}(\widehat{\mathbb{G}})$ then $\theta_{0}$ is simply the extension of the "inclusion" $V N(\tilde{\mathbb{G}}) \supseteq \tilde{\mathbb{G}}=\operatorname{Gr}(\widehat{\mathbb{G}}) \rightarrow L^{\infty}(\widehat{\mathbb{G}})$. In particular, the von Neumann algebra generated by $\operatorname{Gr}(\widehat{\mathbb{G}})$ is isomorphic to $V N(\tilde{\mathbb{G}})$.

The pre-adjoint of $\theta_{0}$ gives us a (complete) quotient map $L^{1}(\widehat{\mathbb{G}}) \rightarrow A(\tilde{\mathbb{G}})$, the Fourier algebra of $\tilde{\mathbb{G}}$. That is, for each $\widehat{\omega} \in L^{1}(\widehat{\mathbb{G}})$ we obtain a function $a: \widetilde{\mathbb{G}}=\operatorname{Gr}(\widehat{\mathbb{G}}) \rightarrow \mathbb{C}, \widehat{u} \mapsto\langle\widehat{u}, \widehat{\omega}\rangle$. Clearly $a$ is continuous; the content of the theorem is that $a \in A(\tilde{\mathbb{G}})$, and that every member of $A(\tilde{\mathbb{G}})$ arises in this way.

The following improves [16, Proposition 5.17], in that we do not need to assume that $\mathbb{G}$ is discrete. We refer to [3] for the notion of a coamenable quantum group.

Theorem 5.6. Let $\widehat{\mathbb{G}}$ be coamenable. Then $\widetilde{\mathbb{G}}$ is amenable.

Proof. That $\widehat{\mathbb{G}}$ is coamenable is equivalent to $L^{1}(\widehat{\mathbb{G}})$ having a bounded approximate identity. As $A(\tilde{\mathbb{G}})$ is a quotient of the Banach algebra $L^{1}(\widehat{\mathbb{G}})$, it follows that $A(\tilde{\mathbb{G}})$ also has a bounded approximate identity, and so $\tilde{\mathbb{G}}$ is amenable, as claimed.

Let us finish by observing the following corollary of the universal property of $\tilde{\mathbb{G}}$, which shows that all "classical" closed quantum subgroups are Vaes closed.

Corollary 5.7. Let $H$ be a locally compact group, identified as a (Woronowicz) closed subgroup of $\mathbb{G}$. Then $H$ is Vaes closed.

Proof. By Theorem 5.3, the morphism $H \rightarrow \mathbb{G}$ factors through a homomorphism $H \rightarrow \tilde{\mathbb{G}}$. To be precise, let the morphism $H \rightarrow \mathbb{G}$ be given by a Hopf $*$-homomorphism $\phi: C_{0}^{u}(\mathbb{G}) \rightarrow C_{0}(H)$, which is surjective by assumption. Let $\theta: C_{0}^{u}(\mathbb{G}) \rightarrow C_{0}(\tilde{\mathbb{G}})$ be the surjective Hopf $*$-homomorphism from Theorem 5.2, so by Theorem 5.3 there is a Hopf $*$-homomorphism $\psi: C_{0}(\tilde{\mathbb{G}}) \rightarrow M\left(C_{0}(H)\right)$ with $\psi \circ \theta=\phi$. As $\theta$ is surjective, it follows that $\psi$ maps into and onto $C_{0}(H)$. Thus $\psi$ identifies $H$ as a closed subgroup of $\widetilde{\mathbb{G}}$.

Equivalently, as explained after Theorem 5.3, we have on the dual side that $C^{*}(H) \rightarrow M\left(C_{0}^{u}(\widehat{\mathbb{G}})\right)$ factors through $C^{*}(H) \rightarrow C^{*}(\tilde{\mathbb{G}})$. By Herz restriction, this map drops to an injective normal $*-$ homomorphism $V N(H) \rightarrow V N(\tilde{\mathbb{G}})$, and by the result above, we have $V N(\tilde{\mathbb{G}}) \rightarrow L^{\infty}(\widehat{\mathbb{G}})$. The composition gives the required injective normal $*$-homomorphism $V N(H) \rightarrow L^{\infty}(\widehat{\mathbb{G}})$.

\section{References}

[1] O. Y. Aristov, Amenability and compact type for Hopf-von Neumann algebras from the homological point of view in Banach algebras and their applications, Contemp. Math. 363 (2004) $15-37$.

[2] S. Baaj, S. Vaes, Double crossed products of locally compact quantum groups, J. Inst. Math. Jussieu 4 (2005) 135-173. 
[3] E. Bédos, L. Tuset, Amenability and co-amenability for locally compact quantum groups, Internat. J. Math. 14 (2003) 865-884.

[4] N. Brown, N. Ozawa, $C^{*}$-algebras and finite-dimensional approximations. American Mathematical Society, Providence, RI, 2008.

[5] J. De Cannière, On the intrinsic group of a Kac algebra, Proc. London Math. Soc. 40 (1980) $1-20$.

[6] H. G. Dales, Banach algebras and automatic continuity. Oxford University Press, New York, 2000.

[7] M. Daws, Completely positive multipliers of quantum groups, Internat. J. Math. 23 (2012) 1250132, 23pp.

[8] M. Daws, Multipliers of locally compact quantum groups via Hilbert $C^{*}$-modules, J. Lond. Math. Soc. 84 (2011) 385-407.

[9] M. Daws, H. Le Pham, Isometries between quantum convolution algebras, Q. J. Math. 64 (2013) 373-396.

[10] M. Daws, P. Kasprzak, A. Skalski, P. Sołtan, Closed quantum subgroups of locally compact quantum groups, Adv. Math. 231 (2012) 3473-3501.

[11] E. G. Effros, Z.-J. Ruan, Operator spaces. Oxford University Press, New York, 2000.

[12] C. Herz, Harmonic synthesis for subgroups, Ann. Inst. Fourier (Grenoble) 23 (1973) 91-123.

[13] Z. Hu, M. Neufang, Z.-J. Ruan, Completely bounded multipliers over locally compact quantum groups Proc. Lond. Math. Soc. 103 (2011) 1-39.

[14] M. Junge, M. Neufang, Z.-J. Ruan, A representation theorem for locally compact quantum groups, Internat. J. Math. 20 (2009) 377-400.

[15] M. Kalantar, Representation of left centralizers for actions of locally compact quantum groups, Internat. J. Math. 24 (2013) 1350025, 10pp.

[16] M. Kalantar, M. Neufang, From quantum groups to groups, Canad. J. Math. 65 (2013) 10731094.

[17] J. Kustermans, Locally compact quantum groups in the universal setting, Internat. J. Math. 12 (2001) 289-338.

[18] J. Kustermans, Locally compact quantum groups in Quantum independent increment processes. I, Lecture Notes in Math. 1865, pp. 99-180. Springer, Berlin, 2005.

[19] J. Kustermans, S. Vaes, Locally compact quantum groups in the von Neumann algebraic setting, Math. Scand. 92 (2003) 68-92.

[20] J. Kustermans, S. Vaes, Locally compact quantum groups, Ann. Sci. École Norm. Sup. (4) 33 (2000) 837-934.

[21] E. C. Lance, Hilbert C-modules. A toolkit for operator algebraists. Cambridge University Press, Cambridge, 1995.

[22] T. Leinster, Basic Category Theory. Cambridge University Press, Cambridge, 2014. 
[23] T. Masuda, Y. Nakagami, S. L. Woronowicz, A $C^{*}$-algebraic framework for quantum groups, Internat. J. Math. 14 (2003) 903-1001.

[24] R. Meyer, S. Roy, S.L. Woronowicz, Homomorphisms of quantum groups, Münster J. Math. 5 (2012), 1-24.

[25] C.-K. Ng, Morphisms of multiplicative unitaries, J. Operator Theory 38 (1997) 203-224.

[26] P. Soltan, S. L. Woronowicz, From multiplicative unitaries to quantum groups. II, J. Funct. Anal. 252 (2007) 42-67.

[27] N. Spronk, Measurable Schur multipliers and completely bounded multipliers of the Fourier algebras Proc. London Math. Soc. 89 (2004) 161-192.

[28] S. Vaes, A new approach to induction and imprimitivity results, J. Funct. Anal. 229 (2005) 317-374.

[29] S. Vaes, Locally compact quantum groups, PhD. thesis, Katholieke Universiteit Leuven, 2001. Available from http://wis.kuleuven.be/analyse/stefaan/

[30] S. Vaes, A. van Daele, Hopf $C^{*}$-algebras, Proc. London Math. Soc. 82 (2001) 337-384.

[31] S. L. Woronowicz, From multiplicative unitaries to quantum groups, Internat. J. Math. 7 (1996) 127-149.

Author's Address: School of Mathematics

University of Leeds

Leeds

LS2 9JT

United Kingdom

Email: matt.daws@cantab.net 